\title{
Characterization of Heat Transfer Enhancement for an Oscillating Flat Plate-Fin
}

\author{
Aevelina Rahman
}

Thesis submitted to the faculty of the Virginia Polytechnic Institute and State University in partial fulfillment of the requirements for the degree of

Master of Science

In

Mechanical Engineering

Danesh K. Tafti

Thomas E. Diller

Rui Qiao

05/06/2020

Blacksburg, Virginia

Keywords: Oscillating flat plate-fin; low Reynolds number; heat transfer enhancement; plunge velocity

Copyright: 2020, International Journal of Heat and Mass Transfer CC BY-NC-ND (CC BY Non-Commercial, No Derivative Works) 


\title{
Characterization of Heat Transfer Enhancement for an Oscillating Flat Plate-Fin
}

\author{
Aevelina Rahman
}

\begin{abstract}
Heat transfer augmentation is of paramount importance in energy transfer and storage systems and the idea of using the inherent vibrations in a system to enhance heat transfer needs to be thoroughly researched upon. The current study numerically investigates an infinitesimally thin plate-fin undergoing forced oscillations over a range of amplitudes and frequencies in the presence of an approach flow. Reduced frequencies of $0.25 \leq k \leq 16$ and plunge amplitudes of $0.03125 \leq h \leq 8$ are investigated at $R e=100$ and $\operatorname{Pr}=0.71$. It is shown that the combined effect of frequency and amplitude on heat transfer enhancement can be accounted for as a single parameter "plunge velocity" $(0.25 \leq k h \leq 4)$ instead of the individual frequency and amplitude values. For $k h>0.5$ a significant increase in Nusselt number $(N u)$ is observed compared to a stationary plate. With increasing $k h$ or more vigorous oscillations, the increase in $N u$ becomes more prominent and similar trends and comparable magnitudes were observed for a constant $k h$ value. Unlike the hydrodynamic counterpart of the study, both Leading Edge Vortices (LEVs) and Trailing Edge Vortices (TEVs) are found to act positively to induce enhanced heat transfer on the plate. Finally, the dependence of heat transfer augmentation on the frequency and amplitude of vibration is quantified with a simple parameterization for a plate-fin in a fluid medium.
\end{abstract}




\title{
Characterization of Heat Transfer Enhancement for an Oscillating Flat Plate-Fin
}

\author{
Aevelina Rahman
}

\section{GENERAL AUDIENCE ABSTRACT}

Heat transfer enhancement is of paramount importance in energy transfer and storage systems. The idea of using the inherent mechanical vibrations in a heat producing system to enhance transfer of unwanted heat from that system needs to be thoroughly researched upon. To investigate this idea, we numerically study an infinitesimally thin plate-fin undergoing forced oscillations over a range of amplitudes and frequencies in the presence of an incoming air flow. It is shown that the combined effect of frequency and amplitude on heat transfer enhancement can be accounted for as a single parameter called "plunge velocity" instead of the individual frequency and amplitude values. For a significant plunge velocity, a significant increase in Nusselt number $(\mathrm{Nu})$ is observed compared to a stationary plate representing an increase in the extent of heat transferred. With more vigorous oscillations, the increase in $N u$ becomes more prominent and similar trends and comparable magnitudes were observed for a constant $k h$ value. Finally, the dependence of heat transfer augmentation on the frequency and amplitude of vibration is quantified with a simple parameterization for a plate-fin in a fluid medium. 


\section{ACKNOWLEDGMENTS}

To start, I would like to thank Almighty ALLAH for giving me the strength, knowledge, ability and opportunity to complete this research satisfactorily. Next, I would take the opportunity to thank my parents and brother for their continual support. I could not have succeeded without their constant encouragement and guidance throughout the years. Also a special gratitude to my husband Ashrarul Haq Sifat for his endless support both academically and mentally. I thank my advisor, Dr. Danesh Tafti, who provided me the opportunity to work on heat transfer aumentation. I am very grateful for the guidance, resources, and advice that have been provided to me by his experience. Thanks as well to Dr. Thomas Diller and Dr. Rui Qiao for lending their time and wisdom to my research and defense. I thank the Advanced Research Computing at Virginia Tech for the computational resources and technical support provided. Thanks to my lab mates in HPCFD lab who helped me with their valuable feedback and suggestions. Finally, I would like to thank Virginia Tech, Mechanical Engineering Department and all the students who came before me for providing this opportunity to work on this amazing and impactful project. 


\section{TABLE OF CONTENTS}

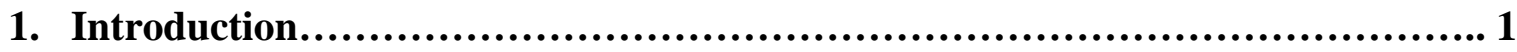

1.1 Active and Passive Methods of Heat Transfer Enhancement............... 1

1.2 Inherent Vibration of Systems and Heat Transfer Enhancement............ 4

2. Governing Equations and Methodology .......................................... 7

3. Computational Geometry and Parametric Range................................ 11

3.1 Computational Domain Specification................................ 11

3.2 Boundary Conditions............................................... 14

3.3 Vibration Motion Specification...................................... 15

3.4 Practical Applicability Range........................................ 16

3.5 Nusselt Number; Characterizes Heat Transfer.......................... 18

4. Results and Discussion........................................................... 19

4.1 Grid Independency Study........................................... 19

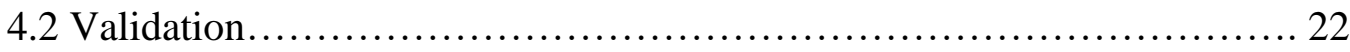

4.3 Heat Transfer Characterization of Vibrating Plate-Fin................... 24

4.3.1 Vortex Dynamics on a Vibrating Fin....................... 24

4.3.2 Vortex Dynamics and Heat Transfer......................... 25

4.3.3 Local Nusselt Number and Plunge Velocity................ 31

4.3.4 Heat Transfer Enhancement Factor.......................... 33

4.3.5 Behavioral Trend of Nusselt Number....................... 34

4.3.6 Energy Advantage of Vibrating Plate-Fin.................. 36

4.4 Parameterization of Nusselt Number.................................... 38

5. Summary and Conclusions................................................... 40

References........................................................................ 42 


\section{Introduction}

\subsection{Active and Passive Methods of Heat Transfer Enhancement}

The increasing emergence of diverse technologies worldwide has increased the global energy consumption exponentially. A large percentage of this energy is in the form of heat and thus enhanced heat transfer mechanisms are at the forefront of energy transfer and storage. Some examples of state of the art enhancement techniques include novel designs for latent heat thermal energy storage (LHTES) devices [1], spray-cooling modules [2], metal foam heat exchangers [3], heat sinks manufactured by selective laser melting [4], technologies involving phase change [5], graphene-based materials [6], Nano-fluids [7] etc. Enhancement techniques can be broadly grouped into two categories; active and passive methods. Systems need some external power to facilitate enhancement with active methods while passive methods require no direct application of external power, rather utilize surface modifications or insertion of swirl devices which change the flow pattern, disturb the thermal boundary layer and consequently facilitate high heat transfer. Artificial roughness in forms of dimples, cavities and corrugations, extended surface as in ribs, fins, treated surfaces and coiled tubes have been extensively studied in the literature as popular passive heat transfer enhancement techniques [8-11]. Guo et al. [12] established the interplay between velocity and temperature field to be an active component for convective heat transfer enhancement. Accordingly displacement enhancement devices or vortex generators are inserted into flow channels to indirectly improve energy transport via vortices that sweep downstream of the element as comprehensively reviewed by Chai et al. [13]. For example, 
conical strip inserts in a circular tube resulted in increased Nusselt number for laminar flow condition [14] while discontinuous helical fins were found to increase turbulent heat transfer in air to water heat exchangers [15]. Alekseenko et al. [16] positively related heat and mass transfer processes with vortex flows. Swirl flow generating devices have been applied in a number of heat exchange scenarios in various forms; in rectangular channels as cylindrical grooves [17], in heat exchanger tubes as discrete inclined grooves [18], in microchannel [19] and mini-channel flows [20], and in pipe and tube flows [21] as wavy-tape inserts. Moreover flow induced vibrations (FIV) impaired on heat exchanging surfaces have also been shown to increase heat transfer by Yakut et al. for induced vibrations from conical rings [22], by Tian et al. for crossflow induced vibration [23] and by Shi et al.[24] for vortices shed from a cylinder. For forced convection heat transfer from a circular cylinder with a flexible fin in laminar flow, Sun et al. [25] showed that when the vortex shedding frequency approached the natural frequency of the fin, the FIV frequency locked on to the natural frequency and the fin exhibited large-amplitude vibration improving convection heat transfer. Also, self-agitators (airfoil-based [26] and caudalfin-inspired [27]) were used to generate vortices to enhance flow mixing and thus heat transfer performance in exchangers operating in laminar-transitional-turbulent regime. All the passive techniques although do not require any external power eventually lead to an increase in fluid pressure drop [28] as they use power from the system itself.

On the other hand, active methods utilize other forms of energy input into the heat exchange device for enhancement. Electro-hydrodynamics and Magneto-hydrodynamics utilizes the effect of electric and magnetic field on the heat transfer fluid $[29,30]$ which increases the motion of fluid leading to disruption of the boundary layer and enhancement of heat transfer. Externally 
imposed vibrations to the heated surface or the working fluid have also been used as an enhancement technique. Vibrations or pulsations to the fluid can range from $1 \mathrm{~Hz}$ to ultrasound [31] and enhance heat transfer significantly. Acoustic streaming caused by high frequency ultrasound intensifies the heat transferred in convection [32] and pool boiling [33].

Vibration of the heated surface at either low or high frequency, has been used in the literature to improve single-phase heat transfer. Experimental investigations were done by Klaczak et al. [34] on the influence of vibration applied in the same direction as flow medium on a horizontal heat exchanger and Forbes et al. [35] on the effect of mechanical vibrations on natural convective heat transfer in an enclosure of rectangular cross-section. Both studies positively correlated the vibration frequency with Nusselt number up to the resonance frequency of the structure. Effect of vibration on free convection has also been tested for heated wires subjected to transverse vibration in air [36], spheres subjected to sinusoidal vibration in the vertical plane [37], cylinders subjected to transverse mechanical vibrations [38], piezo-electrically actuated cantilevers at resonance [39], and vibrating round and annulus [40] tubes. Almost all these studies presented some form of correlation involving vibration frequencies, accelerations and sometimes amplitudes with heat transfer. For forced convection, correlations are available for a sphere [37] and a horizontal cylinder [41] subjected to sinusoidal vibrations perpendicular to inflow with a frequency range of 2.5 to $15.5 \mathrm{~Hz}$ and 3.3 to $46.7 \mathrm{~Hz}$ and a non-dimensional amplitude range of 0.16 to 1.34 and 0.43 to 3.67 , respectively. An analogy of heat and mass transfer was made by Takahashi et al. for the forced convection heat transfer from a vibrating sphere, a cylinder and a square-section tube [42]. 


\subsection{Inherent Vibration of Systems and Heat Transfer Enhancement}

The idea of using the inherent vibrations in a system to enhance heat transfer has also inspired many researchers. This is however, practical only in certain circumstances, as vibrations of sufficient frequency and amplitude to affect the heat transfer may destroy the heat exchanger itself. However, it has been discovered that an acceptable level of vibration, along with damping effects incorporated with the heat sink structure can be allowed to facilitate heat transfer augmentation [43]. In their experimental study, Park et al. [44] attached piezoelectric actuators to the fins of a natural convection air-cooled heat sink and observed increment of the heat transfer coefficient with increasing frequency or increasing amplitude. The best performance was obtained when the system was driven at the resonant frequency which maximized the vibration amplitude. Sarhan et al. [45] experimentally investigated the effect of sinusoidal vertical vibration on thermal performance of a rectangular flat plate under natural convection with a constant heat flux. The amplitude of vibration was varied from 1.5 to $7.5 \mathrm{~mm}$ and the frequency of vibration from 0 to $16 \mathrm{~Hz}$. Micro actuators have also been used to control the vibrations of fins for the purpose of drag reduction in turbulent flows and their contribution to heat transfer [46]. Thus, naturally occurring vibrations could be beneficial to heat transfer and completely eliminating them may not be the best strategy. Rather, harvesting the vibrations up to permissible levels where structural integrity is not compromised and utilizing them to enhance the heat transfer can be beneficial to the overall performance of the heat transfer system.

While a number of studies have investigated the effect of vibrations on heat transfer augmentation, there is no study in the literature which has systematically investigated vibrational 
heat transfer augmentation for a flat plate fin and parameterized this dependence. The two defining characteristics of using vibrations to enhance heat transfer are the frequency and amplitude of vibration. In this study we investigate an infinitesimally thin plate-fin undergoing oscillations over a range of amplitudes and frequencies in the presence of an approach flow. We propose a relationship between the heat transfer enhancement and the frequency and amplitude of vibration by defining a dimensionless reduced frequency ( $k$ ) in Eqn. (1) and a nondimensional amplitude $(h)$ in Eqn. (2) as:

$$
\begin{aligned}
& k=\pi f^{*} c^{*} / U_{\infty}^{*} \\
& h=a^{*} / c^{*}
\end{aligned}
$$

Where,

$f^{*}=$ Frequency of oscillation $(\mathrm{Hz})$

$U_{\infty}^{*}=$ Free-stream velocity $(\mathrm{m} / \mathrm{s})$

$a^{*}=$ Amplitude of oscillation (m)

$c^{*}=$ Plate or chord length $(\mathrm{m})$

The reduced frequency characterizes the ratio of the time scale of the approach flow to the vibrational time scale. We show that the combined effect of frequency and amplitude of vibration can be accounted for as a single parameter given by the "plunge velocity" $(k h)$. Plunge velocity is the average measure of the velocity at which the plate oscillates. This parameter encompasses both the physical effects of frequency (1/time) and amplitude (length). As the plunge velocity increases the average velocity of the leading and trailing edges of the plate also 
increase, thus increasing the strain rate and vortex strength which has a large effect on the flow and heat transfer on the plate surface. It is found in this investigation that as plunge velocity increases the Nusselt number also increases irrespective of the individual values of frequency and amplitude. Thus, the results of this study are significant in quantifying the effect of vibrations on enhancing the heat transfer for a plate-fin in a fluid medium. 


\section{Governing Equations and Methodology}

Space, mass, momentum and energy conservation laws together make up the governing equations for an unsteady incompressible viscous flow in a moving grid coordinate system. The governing equations are solved in a two-dimensional framework with the assumption of constant properties. Non-dimensionalization is done using a suitable reference length scale, velocity scale and temperature scale. The conservative non-dimensional form of the governing equations are written as follows:

Space Conservation Law (SCL):

$$
\frac{\partial}{\partial t}(\forall)-\frac{\partial}{\partial x_{j}}\left(\forall u_{j}^{g}\right)=0
$$

Mass Conservation Law:

$$
\frac{\partial}{\partial x_{j}}\left(\forall u_{j}\right)=0
$$

Momentum Conservation Law:

$$
\frac{\partial}{\partial t}\left(u_{i}\right)+\frac{\partial}{\partial x_{j}}\left(\left[u_{j}-u_{j}^{g}\right] u_{i}\right)=-\frac{\partial}{\partial x_{i}}(p)+\frac{\partial}{\partial x_{j}}\left(\left(\frac{1}{R e}\right) \frac{\partial u_{i}}{\partial x_{j}}\right)
$$

Energy Conservation Law:

$$
\frac{\partial}{\partial t}(T)+\frac{\partial}{\partial x_{j}}\left(\left[u_{j}-u_{j}^{g}\right] T\right)=\frac{\partial}{\partial x_{j}}\left(\left(\frac{1}{R e \cdot P r}\right) \frac{\partial T}{\partial x_{j}}\right)
$$


where,

$\forall=$ cell volume

$u_{i}=$ Cartesian velocity vector

$u_{j}^{g}=$ Grid Cartesian velocity

$T=$ Temperature, and

$p=$ Pressure.

For non-dimensionalization, the reference scale for length and velocity are taken to be the chord length $\left(c^{*}\right)$ and inflow or approach flow velocity $\left(U_{\infty}^{*}\right)$, respectively. Time is nondimensionalized by $\frac{c^{*}}{U_{\infty}^{*}}$ and forces by $\rho^{*} U_{\infty}^{* 2} c^{*}$. The non-dimensional temperature is defined in Eqn. (7).

$$
T=\left(T^{*}-T_{\infty}^{*}\right) /\left(T_{w}^{*}-T_{\infty}^{*}\right)
$$

where the entries with asterisks $\left(^{*}\right)$ depict dimensional values. Reynolds number $(R e)$ and Prandlt number $(P r)$ are defined in Eqns. (8 - 9).

$$
\begin{aligned}
& \operatorname{Re}=U_{\infty}^{*} c^{*} / v^{*} \\
& \operatorname{Pr}=\mu^{*} C_{p}^{*} / \lambda^{*}
\end{aligned}
$$

where,

$T_{\infty}^{*}=$ Inflow temperature in $\mathrm{K}$

$T_{w}^{*}=$ Plate temperature in $\mathrm{K}$

$U_{\infty}^{*}=$ Inflow velocity in $\mathrm{m} / \mathrm{s}$

$c^{*}=$ Chord length in $\mathrm{m}=$ Chord area for $2 \mathrm{D}$

$\rho^{*}=$ Fluid density in $\mathrm{kg} / \mathrm{m}^{3}$ 
$v^{*}=$ Kinematic viscosity in $\mathrm{m}^{2} / \mathrm{s}$

$\mu^{*}=$ Dynamic viscosity in $\mathrm{kg} / \mathrm{ms}$

$C_{p}^{*}=$ Specific heat in $\mathrm{J} / \mathrm{kg} \mathrm{K}$

$\lambda^{*}=$ Thermal conductivity in $\mathrm{W} / \mathrm{mK}$

For this study, $R e=100$ and $\operatorname{Pr}=0.71$ for air.

An in-house body-fitted multiblock incompressible Navier-Stokes Arbitrary Lagrangian Eulerian (ALE) solver, GenIDLEST [47,48] is used for the solution of Eqns. (3 - 6). In the solution algorithm, the grid movement is decoupled from the implementation of fluid conservation laws. At the start of a time step the nodes on the moving boundaries get displaced according to the prescribed motion and the velocity boundary conditions are formulated based on that displacement. This is followed by a rezoning phase during which the movement of the boundary is transmitted to the interior of the domain to obtain the volume grid motion. The boundary motion is first transmitted to the block corners by a series of springs attached to block boundary edges. Then the corner displacement of each block is used to get the new nodal distribution in each block edge, block face, and block volumes, respectively, by using a modified transfinite interpolation (TFI) procedure. The SCL condition (Eqn. 3) is satisfied to get the new grid metrics and the flux due to grid movement. Using a fractional-step method, the mass, momentum and energy conservation laws given by Eqn. (4 - 6) are advanced in time to complete the time step.

A conservative finite-volume discretization is used to discretize the governing equations (Eqns. 3 - 6) on a non-staggered orthogonal grid. Both the convection and diffusion terms in the momentum and energy equations are discretized using a second-order central difference scheme. 
Velocities and pressure are calculated and stored at the computational cell center, whereas fluxes at the cell faces. The time integration is carried out in two steps, a predictor step and a corrector step. In the predictor step an intermediate velocity field is calculated while in the corrector step the intermediate velocity field is updated by solving a pressure equation and satisfying discrete continuity to obtain the velocity at the new time step. The corrector step is advanced in time by using an implicit Crank-Nicolson scheme for the diffusion terms and an explicit second-order Adams-Bashforth scheme for the convection terms. The resulting linear systems are solved by using a pre-conditioned BiCGSTAB method for momentum and pressure. Convergence is achieved when the $\mathrm{L}_{1}$ residual norms fall below $1 \times 10^{-7}$. 


\section{Computational Geometry and Parametric Range}

\subsection{Computational Domain Specification}

The two dimensional (2D) computational domain extends up to 30 chord lengths upstream and 130 chord lengths downstream of the plate-fin of length one chord. The top and bottom boundaries are both located at 30 chord lengths distance from the plate-fin. In effect, the domain is 160 times larger than the flat plate length in the stream wise direction and 60 times larger in the normal direction to ascertain that the boundaries do not have any effect on the flow generated by the oscillating plate. The oscillating plate is initially located at $y=0$ between $x=0$ and 1 . In theory, the plate has zero thickness as the boundary conditions are directly applied to the plate location of $y=0$. A total of 2,173,600 computational cells are used to discretize the domain. Figure 1 shows the variation of the grid spacing with relative position in the domain. The grid spacing is uniform in the stream wise direction from 2 plate lengths upstream to 5 plate lengths downstream. In the normal direction the uniformly spaced region starts from 1.8 plate lengths below the initial position of the plate and extends up to 1.8 plate lengths above the plate. Also, the grid is finest in the region surrounding the plate to accurately resolve the vortices created by the plunging motion. Beyond this uniformly spaced region, the grid is coarsened gradually to balance computational cost. As a moving grid method is implemented, this fine region oscillates with the plate ensuring that the vortices created in the vicinity of the plate are resolved with high precision. In the fine grid region near the plate, the grid spacing is such that there are 
approximately 150 cells in one boundary layer thickness approximated by the Blasius solution at $x=0.5$.

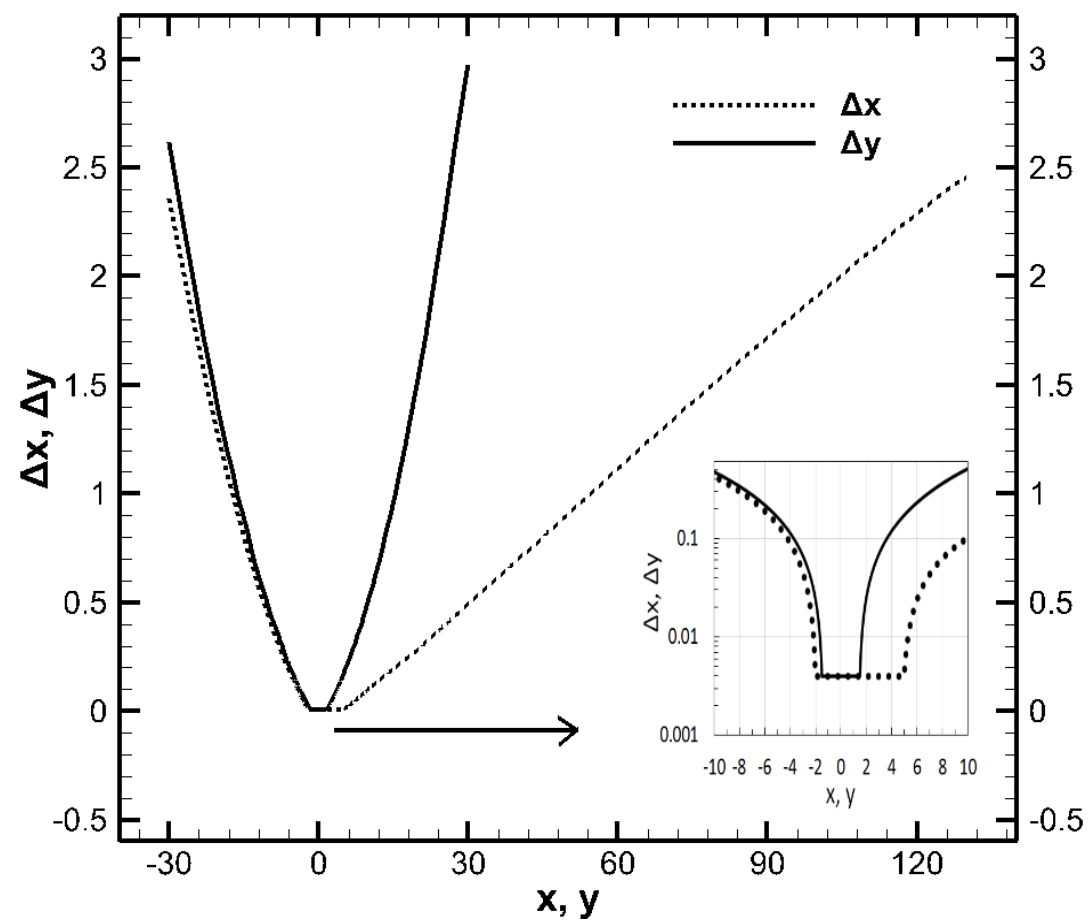

Figure 1: Grid spacing in both stream wise $(x)$ and normal $(y)$ direction. Inset shows uniform grid spacing along the plate in both stream wise and normal direction

Figure 2 shows the z-vorticity $(\omega)$ of a flow field containing some of the most energetic vortex structures $(k=12 ; k h=4)$. As evident from Figure 2, the vortex structures are contained well within the domain and significantly far from all four boundaries ascertaining that the boundary conditions do not influence the evolution of these structures near the plate. Also, when zoomed in very close to the plate $\left(2^{\text {nd }}\right.$ inset of Figure 2), sufficient number of grid cells are present in each vortex core. 


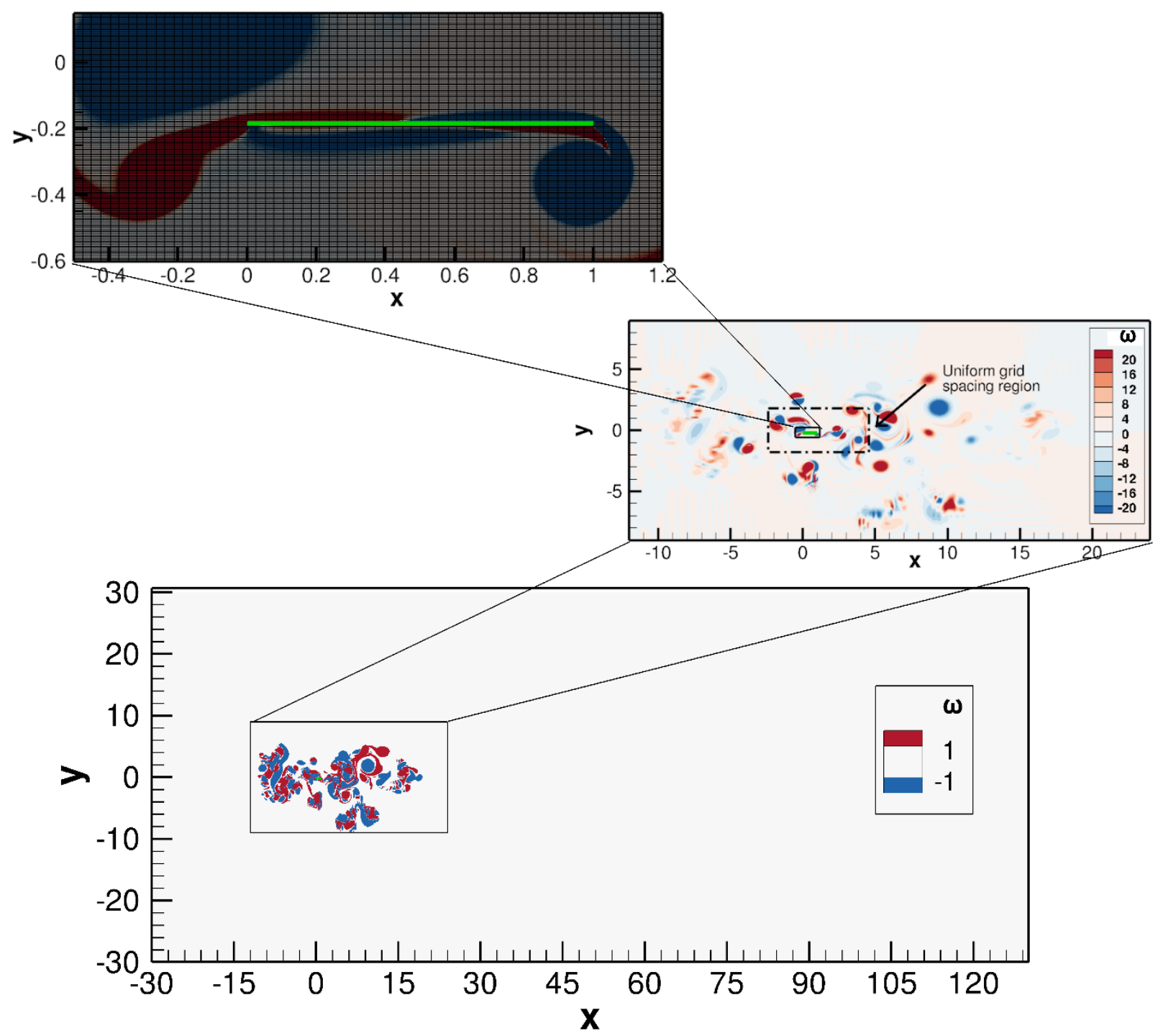

Figure 2: Instantaneous vorticity field (z-vorticity $(\omega))$ at $k h=4$. Insets show the extent of the vortices generated by the plate relative to the size of the domain and to the size of the grid spacing 


\subsection{Boundary Conditions}

The upstream boundary of the domain $(x=-30)$ is set at a non-dimensional inlet velocity of unity. Outflow zero gradient conditions $\left(\frac{d \phi}{d x}=0 ; \phi=u, v, p\right)$ are applied at the downstream boundary $(x=130)$. Boundary conditions $\left(\frac{d \phi}{d y}=0, \phi=u, p \& v=0\right)$ are applied at the top and bottom $y$-directional boundaries of the domain $(y= \pm 30)$. the oscillating plate-fin is treated as a no-slip, no-penetration wall $\left(\vec{u}=0 ; \frac{d p}{d y}=0\right)$. To study the heat transfer on the vibrating plate-fin, a constant temperature boundary condition has been applied on both surfaces of the plate. Based on Eqn. (7), the plate surface is set to $T=1$ and the calculation domain and incoming flow to $T=0$ 


\subsection{Vibration Motion Specification}

The plate-fin oscillates in a pure plunging motion which is governed by a sinusoidal equation

(Eqn. 10). Initially the plate is located at $y=0$ and the plunging motion starts with the plate moving upwards.

$$
Y^{*}=Y_{0}^{*}+a^{*} \sin \left(2 \pi f^{*} t^{*}\right)
$$

where,

$$
\begin{aligned}
& Y^{*}=\text { position of the plate at any time } t(\mathrm{~m}) \\
& Y_{0}^{*}=\text { Initial position of the plate }=0(\mathrm{~m}) \\
& a^{*}=\text { Plunge amplitude }(\mathrm{m}) \\
& f^{*}=\text { Plunge frequency }(\mathrm{Hz}) \\
& t^{*}=\text { time }(\mathrm{s})
\end{aligned}
$$

To maintain orthogonality of the grid throughout the plunge cycle the full extent of a grid line $(-30 \leq x \leq 130)$ (versus only $0 \leq x \leq 1)$ oscillates with the plate. 


\subsection{Practical Applicability Range}

Table 1 summarizes the different combinations of reduced frequency, $k$ (Eqn. 1) and different plunge amplitudes, $h=a^{*} / c^{*}$ (Eqn. 2) simulated in the present study. The investigation is conducted over a wide range of reduced frequency $(0.25 \leq k \leq 16.0)$ and plunge amplitude $(0.03125 \leq h \leq 8.0)$. The flat plate was oscillated with reduced frequencies of $0.25,0.5 .1,2,4,8$, 10, 12 and 16 with amplitudes ranging from 8 to 0.03125 for all the frequencies. This produced a working range of plunge velocity between $0.25 \leq k h \leq 4$. The computational time increases as $k h$ increases and the increase is more significant with increase in amplitude than with frequency increase. It ranges from 2 hours of wall-clock time per oscillation cycle for $k h=1(k=16, h=$ $0.0625)$ to the most compute intensive case for $k h=4(k=1$ and $h=4)$ which takes 52 hours, on 20 Haswell 2xE5 2.5 Ghz cores.

\section{Table 1: Summary of calculations and time-averaging}

\begin{tabular}{llll}
\hline$k$ & $h$ & $\begin{array}{l}\text { Cycles considered in time } \\
\text { avaraging }\end{array}$ & \\
\hline 0.25 & $1.0,2.0,4.0,8.0$ & $9-16$ & \\
0.5 & $0.5,1.0,2.0,4.0$ & $9-16$ & \\
1 & $0.25,0.5,1.0,2.0,4.0$ & $9-16$ & \\
2 & $0.125,0.25,0.5,1.0,2.0$ & $9-16$ & \\
4 & $0.0625,0.125,0.25,0.5,1.0$ & $9-16$ & \\
8 & $0.0625,0.125,0.25,0.5$ & $9-16$ & \\
10 & $0.05,0.1,0.2,0.4$ & $11-20$ & \\
12 & $0.04167,0.083,0.167,0.333$ & $13-24$ & \\
16 & $0.03125,0.0625,0.125,0.25$ & $17-32$ & \\
\hline
\end{tabular}

When traced back to dimensional values, the study covers a wide range of frequency and amplitudes representing a variety of fin applications. For example, a chord length (which is the 
plate length in current study) of millimeter scale corresponds to an effective inflow velocity of $1.5 \mathrm{~m} / \mathrm{s}$ in air for the considered Reynolds number while the various $k$ tested renders a physical frequency range of 400 to $7000 \mathrm{~Hz}$ and thus falls into the regime of typical velocity and frequency range of micro-fin arrays [46]. Likewise, a centimeter range chord length would yield an effective velocity and frequency range that comes out to be compatible with general louver fins [49] and plate fins [50]. The shaded area of Figure 3 encompasses the applicable range of current study. The dashed line is indicative of the effective inflow velocity range for which current study should be applicable.

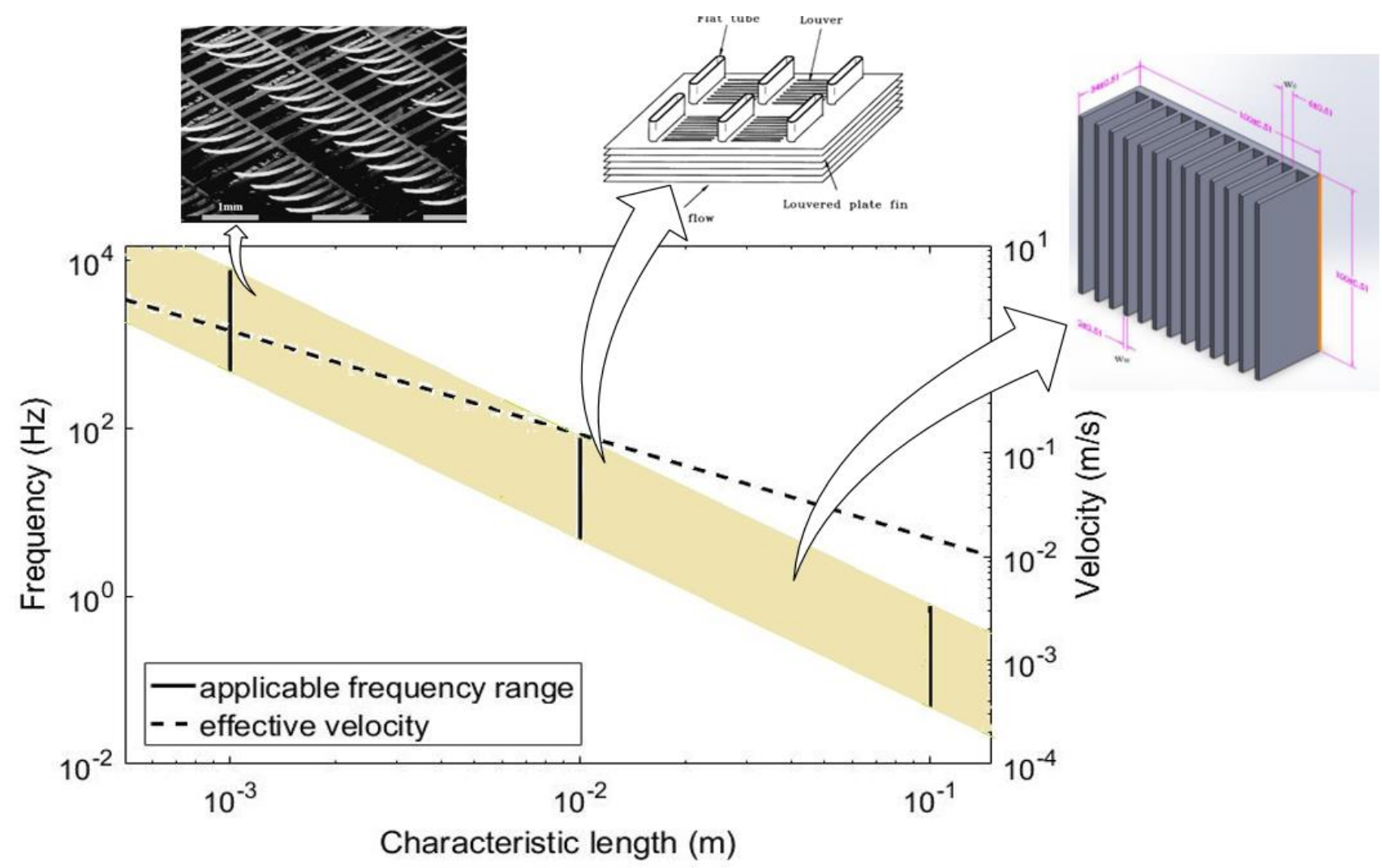

Figure 3: Area of applicability of present study; shaded area depicts the frequency range for chosen characteristic length $(=$ chord $=$ plate length); dashed line indicates the effective velocity range for the approach flow; different fin pictures depict the characteristic lengths, velocities and amplitudes of respective fin arrangements 


\subsection{Nusselt Number; Characterizes Heat Transfer}

The heat transfer performance is characterized by the instantaneous local Nusselt number, $N u_{x}=h_{x}^{*} \times x^{*} / \lambda$ and the instantaneous spatially averaged Nusselt number, $N u_{L}=h_{L}^{*} \times c^{*} / \lambda$, where $h_{x}^{*}$ is the local heat transfer coefficient at a distance $x$ from the leading edge of the plate, and $h_{L}^{*}$ is the mean heat transfer coefficient over the length of the plate. The time-averaged

Nusselt numbers are denoted by $\overline{N u}_{x}=\bar{h}_{x}^{*} x^{*} / \lambda$ and $\overline{N u}_{L}=\bar{h}_{L}^{*} \cdot c^{*} / \lambda$, where the overbar denotes time-averaging.

The local $N u$ number is calculated from the dimensionless calculated temperature distribution by coverting the dimensional definitions of heat transfer coefficient (denoted by $*$ ) as follows:

$$
q^{\mathrm{m} *}=-\left.K^{*} \frac{d T^{*}}{d y^{*}}\right|_{w}=h^{*}\left(\mathrm{~T}_{w}^{*}-\mathrm{T}_{\infty}^{*}\right)
$$

$\left.\frac{d T^{*}}{d y^{*}}\right|_{w}$ is the temperature gradient along the normal direction of the plate, and $q^{\mathrm{m} *}$ is the heat flux. The temperature gradient in Eq. 11 is non-dimensionalized in Eq. 12 as

$$
q^{m *}=-\left.K^{*} \frac{d T}{d y}\right|_{w} \cdot \frac{\left(T_{w}^{*}-T_{\infty}^{*}\right)}{c^{*}}=h^{*}\left(T_{w}^{*}-T_{\infty}^{*}\right)
$$

Multiplying Eq. 12 by $\frac{c^{*}}{\left(T_{w}^{*}-T_{\infty}^{*}\right) x^{*}}$ gives.

$$
\frac{c^{*}}{\left(T_{w}^{*}-T_{\infty}^{*}\right) x^{*}} \times q^{\mathrm{m} *}=-\left.\frac{d T}{d y}\right|_{\text {wall }}=\frac{h^{*} l_{r e f}^{*}}{x^{*}}
$$

which can be written in terms of dimensionless variables obtained from the calculation as:

$$
q^{\prime \prime}=-\left.\frac{d T}{d y}\right|_{\text {wall }}=N u
$$




\section{Results and Discussion}

\subsection{Grid Independency Study}

Because of the strong non-linearity in the flow resulting from vortex-vortex interactions as $k h$ increases, an extensive grid independency study has been conducted. To establish grid independency, six grid levels were tested. The details of the six grids are shown in Table 2. Not only were the number of grid points varied but different grid distribution strategies were also investigated.

Table 2: Details of grid independency study

\begin{tabular}{|c|c|c|c|c|c|c|}
\hline & Grid 1 & Grid 2 & Grid 3 & Grid 4 & Grid 5 & Grid 6 \\
\hline $\begin{array}{l}\text { Number of grid } \\
\text { Points along the } \\
\text { plate length }\end{array}$ & 100 & 132 & 200 & 250 & 250 & 300 \\
\hline $\begin{array}{l}\text { Total number of } \\
\text { cells (millions) }\end{array}$ & 0.14 & 0.25 & 0.56 & 1.77 & 2.18 & 2.97 \\
\hline $\begin{array}{l}\text { Remark } \\
\text { UGS = Uniform } \\
\text { Grid Spacing }\end{array}$ & $\begin{array}{l}\text { UGS } \\
0<x<1\end{array}$ & UGS $0<x<1$ & UGS $0<x<1$ & UGS $2<x<5$ & $\begin{array}{l}\text { UGS } \\
2<x<5 \\
-1.8<y<1.8\end{array}$ & $\begin{array}{l}\text { UGS } \\
2<x<5 \\
-1.8<y<1.8\end{array}$ \\
\hline $\begin{array}{l}\text { Remark } \\
\text { GGC = Gradual } \\
\text { Grid Coarsening }\end{array}$ & $\begin{array}{l}\text { GGC } \\
\text { starting } \\
\text { from the } \\
\text { plate in } y \\
\text { direction }\end{array}$ & $\begin{array}{l}\text { GGC } \\
\text { starting } \\
\text { from the } \\
\text { plate in } y \\
\text { direction }\end{array}$ & $\begin{array}{l}\text { GGC } \\
\text { starting } \\
\text { from the } \\
\text { plate in } y \\
\text { direction }\end{array}$ & $\begin{array}{l}\text { GGC } \\
\text { starting } \\
\text { from the } \\
\text { plate in } y \\
\text { direction }\end{array}$ & $\begin{array}{l}\text { No GGC in the } \\
\text { vicinity of the } \\
\text { plate }\end{array}$ & $\begin{array}{l}\text { No GGC in the } \\
\text { vicinity of the } \\
\text { plate }\end{array}$ \\
\hline
\end{tabular}

To test the grid independency, some representative cases from the three largest plunge velocity values ( $k h=1,2$ and 4$)$ were simulated using all six grids. Figure 4 plots the time-averaged distribution of local Nusselt number $\left(\overline{N u}_{x}\right)$ on the plate for the different grid resolutions. At $k h=$ 
1, the flow is not very energetic, and the results are grid insensitive and all grids produce the same local distribution of Nusselt number. In this case all vortices convect parallel to the plate in the fine grid region and are thus well-resolved. As $k h$ increases, the energy imparted to the flow by the motion of the plate is high which gives rise to strong vorticity production. As shown in Figure 2 the strong vorticity induced velocity fields convect vortices from near the confines of the plate-fin upstream before convecting them back to affect the heat transfer at the plate. Additionally, as $k h$ increases, vortices shed at the trailing edge of the plate, instead of convecting downstream into the wake, are induced onto the plate near the trailing edge resulting in strong chaotic vortex-vortex interactions in this region. These dynamics manifest as greater grid sensitivity at $k h=2$ and 4 near the trailing edge of the plate. This is reflected in Figure 4 in which different grid resolutions and distribution impact the local Nusselt number. Focusing on Grids- 4, 5 and 6, at $k h=2, \overline{N u}_{x}$ is quite independent of the three grids, whereas for $k h=4$, as the grid gets finer, $\overline{N u}_{x}$ decreases in the latter half of the plate.
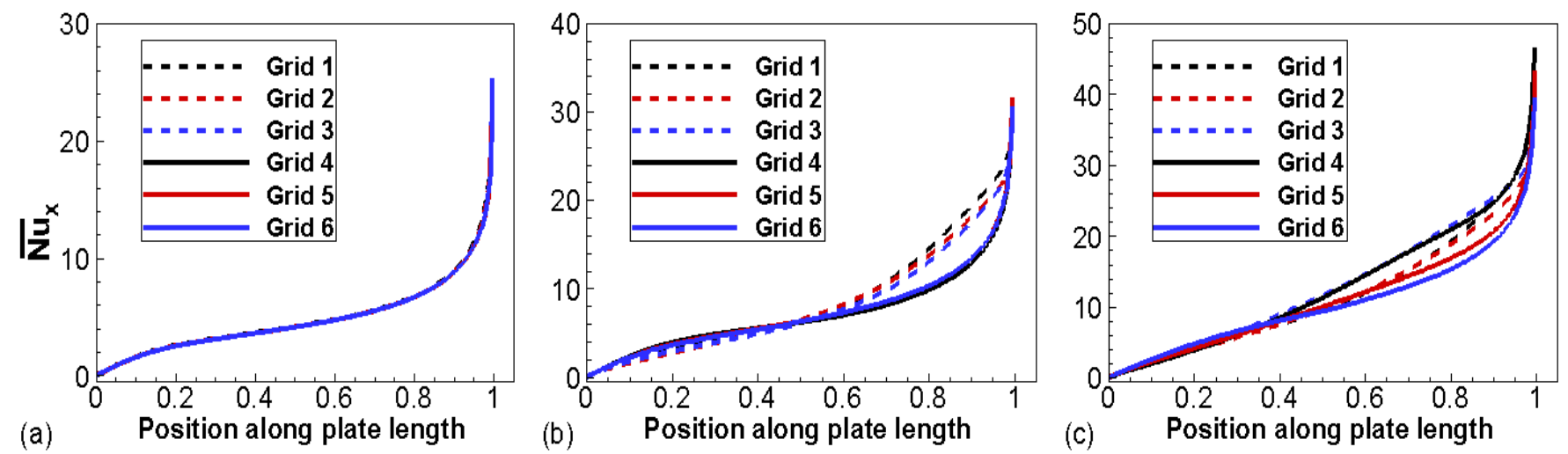

Figure 4: Time averaged Nusselt number considered versus the entire length of the plate $\left(\overline{N u}_{x}\right)$ for three representative cases of different $k h$ on different grids. (a) $k h=1 ; k=8, h=0.125$, (b) $k h$ $=2 ; k=10, h=0.2 \&$ (c) $k h=4 ; k=12, h=0.333$ 
Table 3 compares the grid convergence based on the time and space-averaged Nusselt number $\left(\overline{N u}_{L}\right)$. The percentage difference of mean global Nusselt number $\overline{N u}_{L}$ on Grid-5 in comparison with the finest grid (Grid-6) is within 1\% and of Grid-4 within 5\% of Grid-6 as shown in Figure 5. Thus, Grid-5 is chosen as the base grid on which all results are presented.

Table 3: Comparison of results among six different grid resolutions

\begin{tabular}{lllllllll}
\hline & & & Grid-1 & Grid-2 & Grid-3 & Grid-4 & Grid-5 & Grid-6 \\
\hline & $k$ & $h$ & $\overline{N u}_{L}$ & $\overline{N u}_{L}$ & $\overline{N u}_{L}$ & $\overline{N u}_{L}$ & $\overline{N u}_{L}$ & $\overline{N u}_{L}$ \\
$\boldsymbol{k h}=\mathbf{1}$ & 8 & 0.125 & 10.720 & 10.608 & 10.659 & 10.641 & 10.639 & 10.638 \\
$\boldsymbol{k h}=\mathbf{2}$ & 10 & 0.2 & 16.437 & 16.199 & 15.859 & 15.543 & 14.966 & 14.814 \\
$\boldsymbol{k h}=\mathbf{4}$ & 12 & 0.333 & 24.369 & 23.715 & 22.339 & 22.144 & 21.909 & 21.833 \\
\hline
\end{tabular}

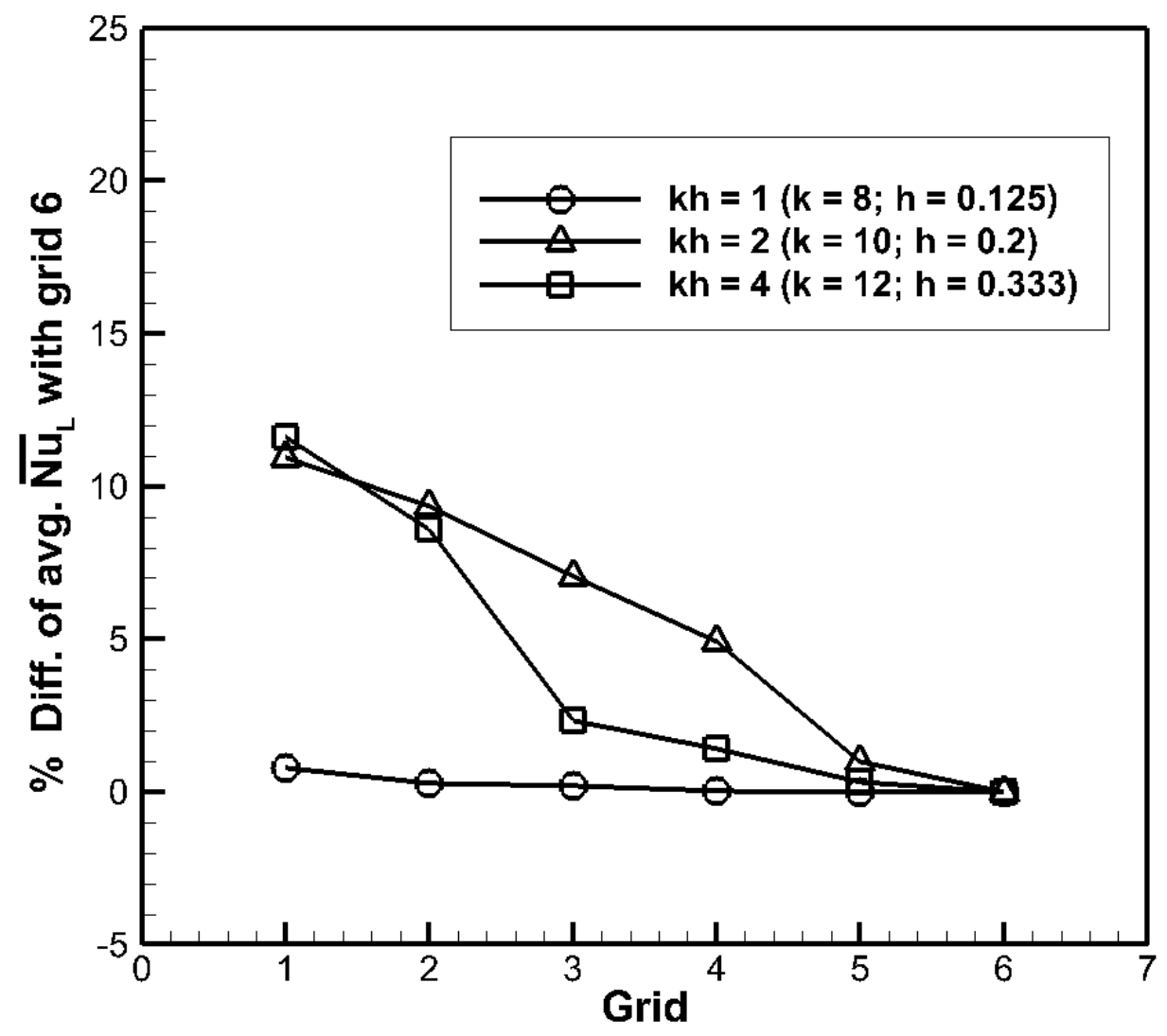

Figure 5: Grid convergence for different $k h$ values 


\subsection{Validation}

We further validate the grid distribution by comparing the distribution of time averaged local

Nusselt number $\left(\overline{N u}_{x}\right)$ along the length of the stationary plate from the numerical simulation with the theoretical values based on the Blasius solution (Eqn. 15) in Figure 6.

$$
\overline{N u}_{x}=0.332 \operatorname{Re}_{x}^{1 / 2} \operatorname{Pr}^{1 / 3}
$$

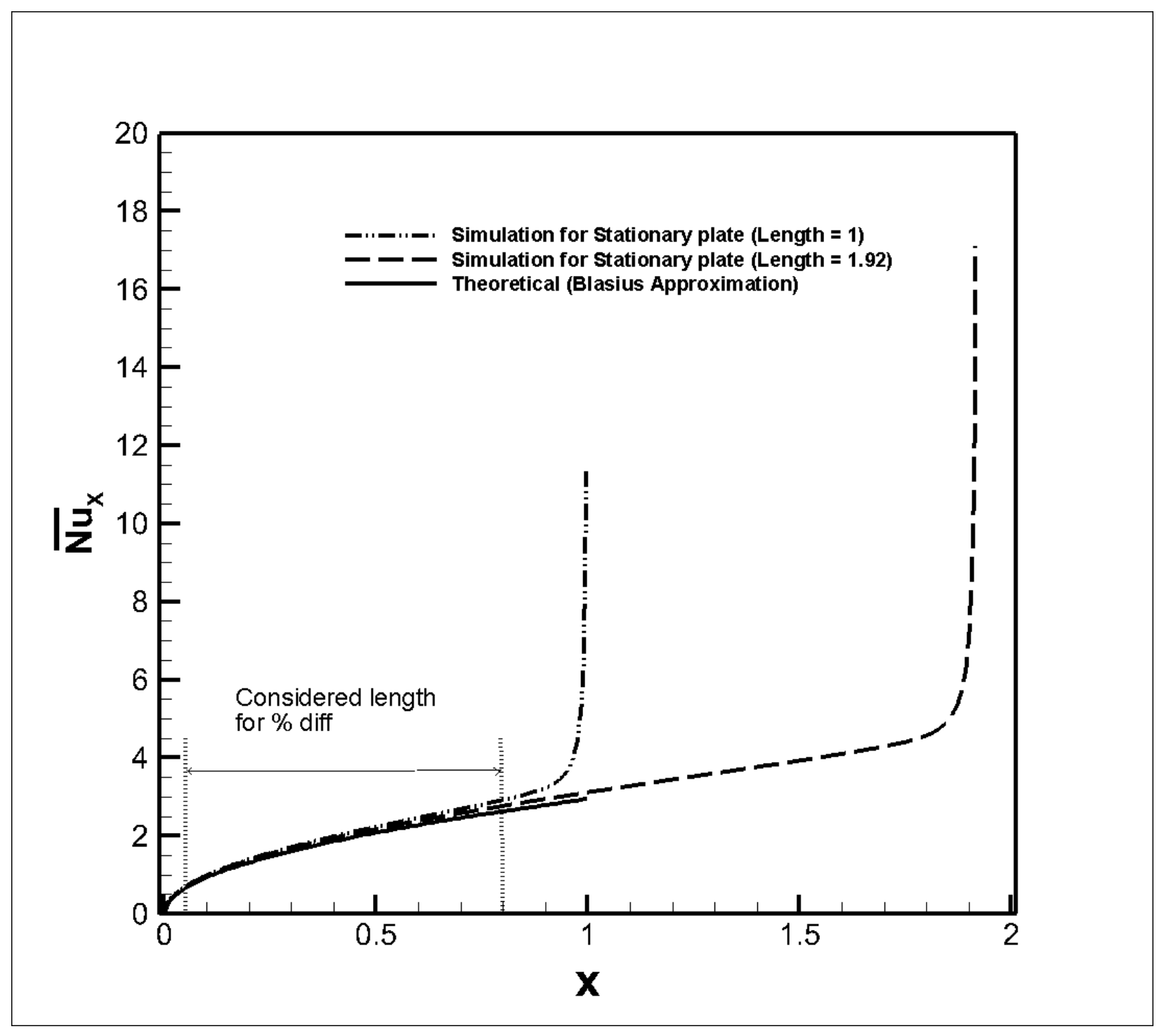

Figure 6: Local Nusselt number validation against Blasius approximation 
An underlying assumption in the Blasius solution is that the plate is infinitely long with no end effects. However, in the finite plate geometry considered, end effects affect the solution upstream of the trailing edge. As the flow approaches the trailing edge it accelerates sharply resulting in an abrupt thinning of the boundary layer thickness near the trailing edge of the plate which gives rise to the sudden rise in the Nusselt number. Moreover, the boundary layer approximation of Blasius does not hold very well when approaching the leading edge at which the boundary layer assumption is not valid. In order to avert the ambiguities of the starting and end effects of the plate, we considered $5 \%$ to $80 \%$ of the plate length for validating with the Blasius solution. In this range, the numerical solution for the stationary plate of non-dimensional length of unity displayed good comparison with Blasius solution with only $7.1 \%$ mean deviation. By eliminating end effects by using a longer plate (non-dimensional length 1.92), this deviation reduces to $4.5 \%$. Considering the fact that in the numerical study, end effects influence the solution in the interior, the mean deviation of $4.5 \%$ is acceptable. 


\subsection{Heat Transfer Characterization of Vibrating Plate-Fin}

\subsubsection{Vortex Dynamics on a Vibrating Fin}

The effect of reduced frequency $(k)$ and plunge amplitude $(h)$ on vortex structure shed from an oscillating plate-fin is discussed in brief. An upward motion of the plate creates a counterclockwise vortex at the leading edge (LEV- leading edge vortex) and a clockwise vortex at the trailing edge (TEV- trailing edge vortex) on the bottom side of the plate. Likewise, a downward motion creates a clockwise LEV and a counterclockwise TEV on the top side of the plate. The extent to which these vortices are attached to the plate during the duration of the plunge cycle determines whether a net thrust or a net drag will result as well as the degree of heat transfer augmentation that is to occur. 


\subsubsection{Vortex Dynamics and Heat Transfer}

The production of thrust or drag is only dependent on shear forces acting on the plate. Thrust is obtained if the vortex induced velocities tend to push or pull the plate in a direction opposing the approach flow for a majority of time in the cycle. Whereas, drag results if the vortices force the plate in the same direction as the inflow. An example of LEV induced negative shear stress and net instantaneous thrust production is shown in Figure 7 on the top surface of the plate for a moderately high $k h$ value of $2(k=16, h=0.125)$. The LEV induces negative velocity (shown by the black line) near the plate surface and the resulting negative shear acts to push the plate up stream. Thus, leading edge vortices (LEVs) contribute towards thrust while trailing edge vortices (TEVs) towards drag as surmised from Figure 7 where LEVs and TEVs are represented by zvorticity $(\omega)$ contours.

However, unlike the hydrodynamics, both LEVs and TEVs act towards increasing the heat transfer from the plate to the surrounding fluid. The continuous formation and shedding of vortices, regardless of them being LEVs or TEVs, consistently entrain cooler fluid to the plate and take away some portion of the energy from the plate. As a pair of vortices shed, fresh cooler fluid comes in the vicinity of the plate and thus heat transfer increases in the presence of a vortex. Figure 7 shows larger gradients of temperature $(T)$ at the leading and trailing edge of the plate $(x=0.01$ and 0.99$)$ whereas the mid positions $(x=0.3,0.5$ and 0.7$)$ have lower temperature gradients as shown by the temperature profiles in pink. This signifies a greater amount of heat transfer in the positions $(x=0.01$ and 0.99$)$ where the LEV and TEV are generated. 


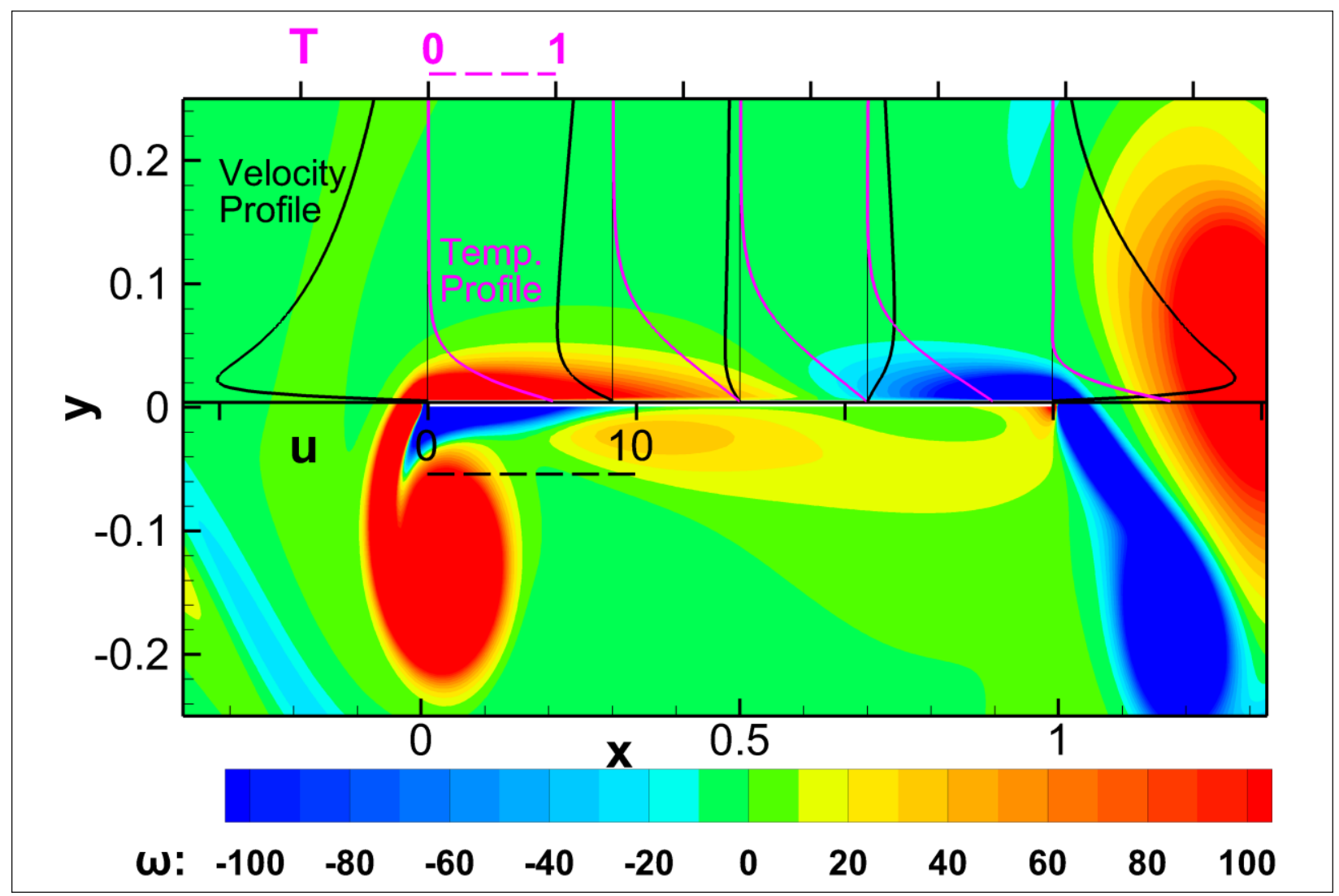

Figure 7: Instantaneous temperature profiles $(T)$ and $u$-velocity profiles $(u)$ at different chord positions ( $x=0.01,0.3,0.5,0.7$ and 0.99 ) in the presence of LEV and TEV shown in terms of $z^{-}$ vorticity $(\omega)$. LEVs induce negative velocity gradients (depicted by black lines) adjacent to the plate (e.g. at $x=0.01,0.3$ and 0.5 ) to produce thrust while TEVs induce positive velocity gradients (depicted by black lines) adjacent to the plate (e.g. at $x=0.7$ and 0.99 ) to produce drag. Both LEV and TEV induce strong positive temperature gradients (depicted by pink lines) to enhance heat transfer (e.g. at $x=0.01$ and 0.99 ) whereas the temperature gradients at mid-plate (e.g. at $x=0.3,0.5$ and 0.7 ) are less steep indicating less heat transfer than that at leading and trailing edge. The dashed pink and black lines, respectively, denote the temperature $(T)$ and $u-$ velocity $(u)$ scale used in the figure.

Figure 8 shows another representative case where the plate is oscillated with $k=1$ and $h=1$ giving a plunge velocity, $k h$ of 1 . The color contour shows the temperature distribution near the plate and the line contour plots the coherent vorticity. As we can see, the temperature contour closely follows the coherent vortex structures which drives the enhancement of heat transfer. 
At the start of the down stroke a negative clockwise LEV starts forming on the top surface of the plate which grows during the downstroke and sheds at the end. A similar cycle is evident for the LEV that starts forming at the bottom surface at the beginning of the upstroke which finally sheds into the wake at the end of the upstroke. In spite of the presence of asymmetries in vortex generation and shedding, a close relationship is observed between the coherent vortices and the temperature contour throughout the entire stroke. At the beginning of the downstroke, with the shed LEV at the bottom surface from the previous upstroke the thermal boundary layer is thickest and the heat transfer or Nusselt number is at its minimum. As the LEV on the top surface of the plate grows during the downstroke, the thermal boundary layer becomes thinner. The heat transfer increases due to the high temperature gradients in the thinner thermal boundary layer and becomes maximum at the end of downstroke and beginning of upstroke. During the upstroke, the LEV already shed from top surface and the incipient one from the bottom surface tend to trap the heated fluid into close proximity of the plate. Hence the thermal boundary layer again starts to become thick and thus $\mathrm{Nu}$ decreases comparatively.

Although the $N u$ variation in a plunge cycle goes through periods of augmentation and attenuation, there is a net increment of $N u$ and overall heat transfer when compared to the stationary plate-fin. Figure 9 shows the time averaged temperature field of a stationary plate-fin in comparison with three other cases of increasing plunge velocities. The stationary plate has the thickest thermal boundary layer around it with a strong thermal wake region giving rise to low temperature gradients at the surface. Also, once the flow-field around the stationary plate is established, the heated fluid/air surrounding it will exist indefinitely and there is no further opportunity for cooler air to come in the vicinity of the plate to increase heat transfer. But when the plate-fin is oscillating with some frequency and amplitude, continuous generation and 
shedding of vortex structures take away the heated fluid from its vicinity and entrain cooler fluid. This continuous replenishment of cooler fluid in the proximity of the plate-fin surface increases the heat transfer. The larger the scale of vibration/oscillation, the greater amount of heat transfer is observed. As Figure 9 shows, with increasing plunge velocity, the time averaged thermal boundary layer around the plate-fin becomes thinner which is indicative of higher temperature gradients and thus higher heat transfer. 


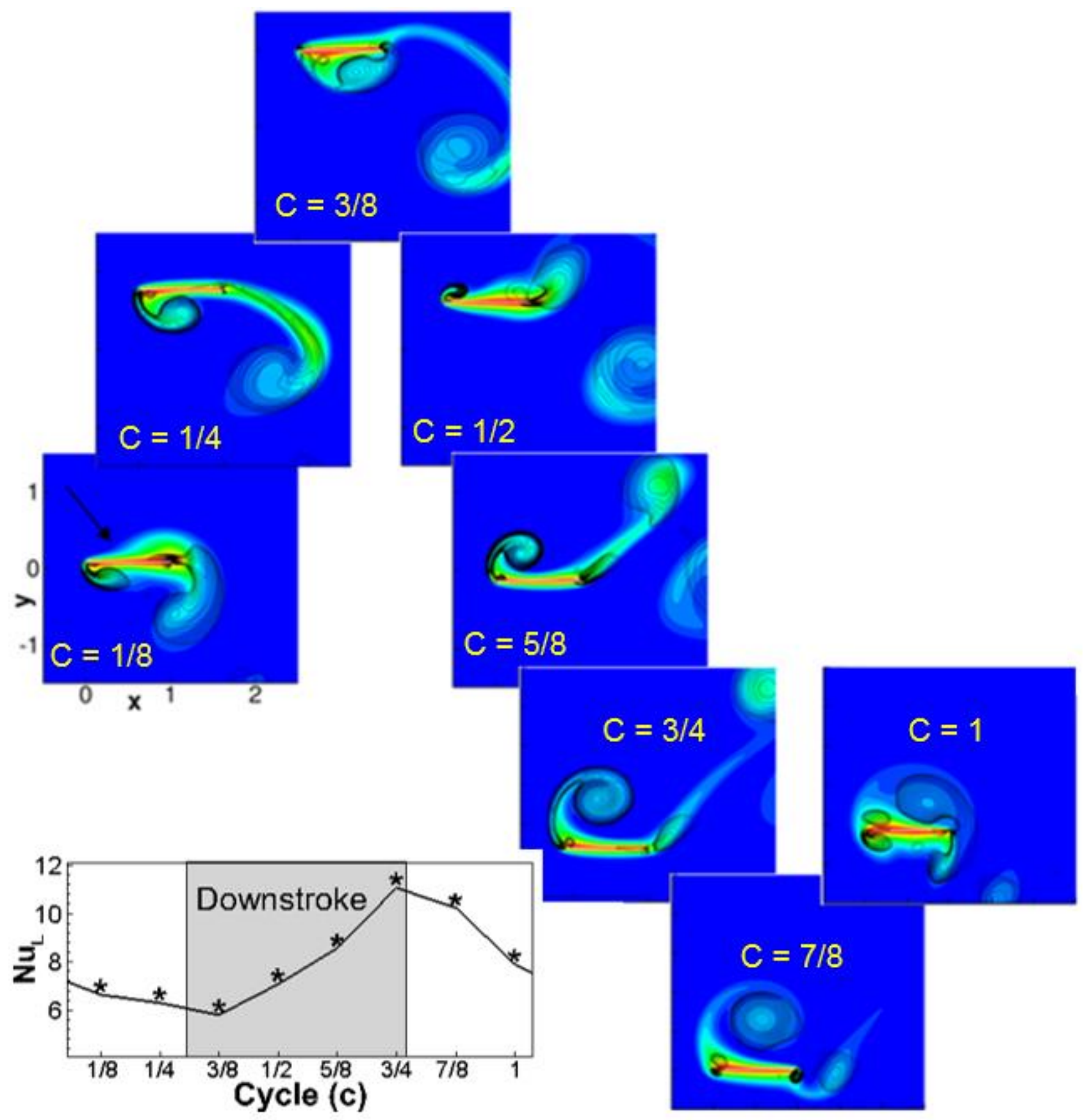

T: $\begin{array}{lllllllllllllllll}0.1 & 0.15 & 0.2 & 0.25 & 0.3 & 0.35 & 0.4 & 0.45 & 0.5 & 0.55 & 0.6 & 0.65 & 0.7 & 0.75 & 0.8 & 0.85 & 0.9\end{array}$

Figure 8: Temperature and vorticity contours near the plate with instantaneous $N u_{L}$ for the shown cycle for a representative case of $k=1$ and $h=1$ giving a plunge velocity $k h=1$; color contour shows the temperature distribution near the plate whereas line contours show the coherent vortex structures. The entire cycle $(c=1)$ is broken down into 8 instances and represented by the 8 rectangular snapshots. 


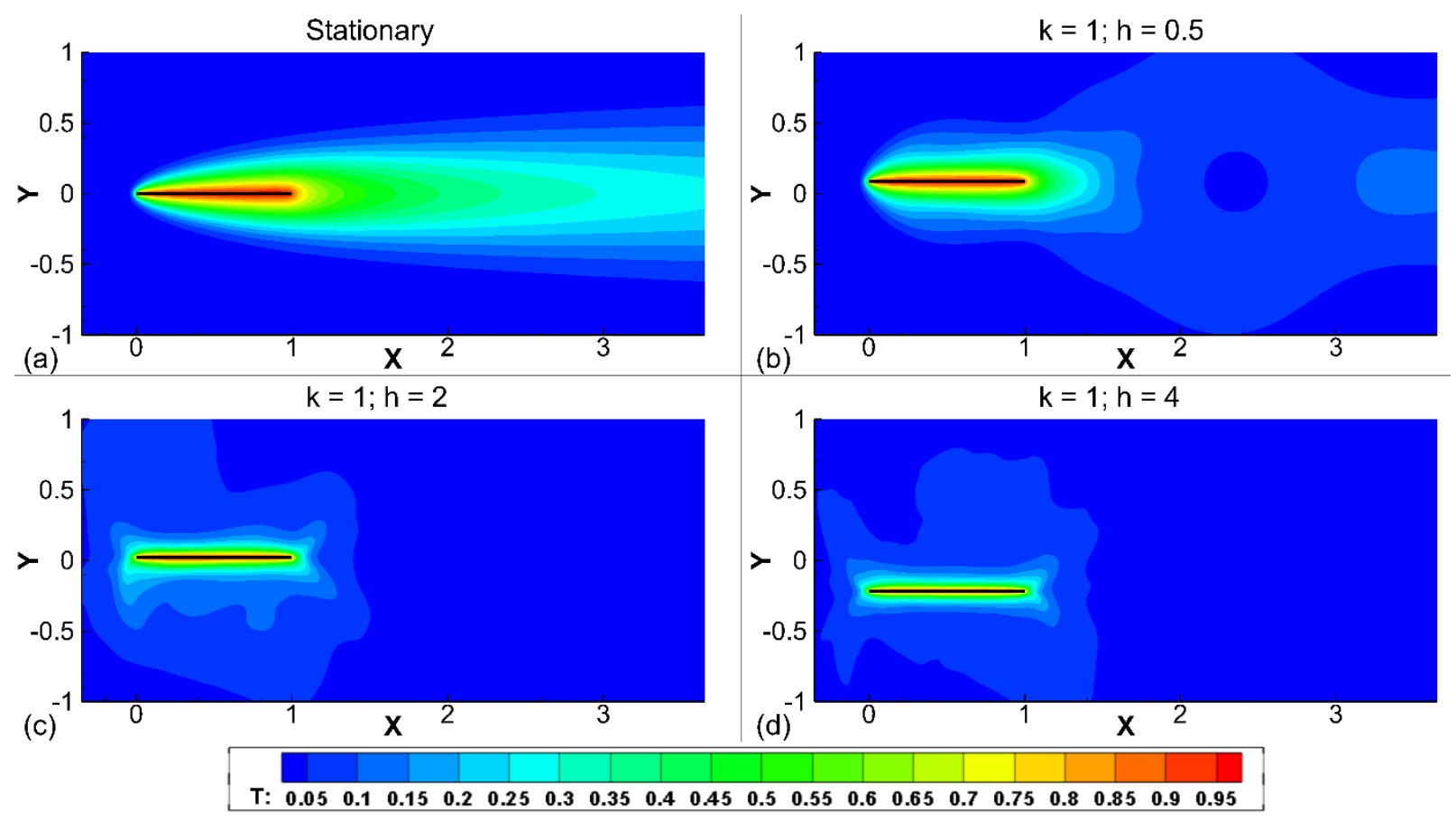

Figure 9: Time averaged temperature field of a stationary plate in comparison with three cases of increasing plunge velocities. (a) $k h=0$, (b) $k h=0.5$, (c) $k h=2$ and (d) $k h=4$ 


\subsubsection{Local Nusselt Number and Plunge Velocity}

Figure 10 shows the $\overline{N u}_{x}$ distribution along the plate length for the five different plunge velocity $(k h=0.25,0.5,1,2$ and 4$)$ values that are tested in the current study. For $k h \geq 0.5$ there is a significant increase in $\overline{N u}_{x}$ distribution over the entire length of the plate compared to the stationary plate. As the oscillations become more vigorous, i.e. the plate-fin vibrates with higher frequency ( $k$ increasing) or amplitude ( $h$ increasing), the increase in $N u$ becomes more prominent. It is noteworthy that the Nusselt number only shows a strong dependence on $k h$. irrespective of the individual values of $k$ and $h$. For a particular $k h$ the $\overline{N u}_{x}$ distribution along the plate-fin length follow the same trend and have comparable magnitude. For example, plunge velocity $k h=1$ was achieved with 9 different combinations of frequency and amplitude. In spite of $k$ and $h$ individually varying over a factor as high as 64, the distribution and magnitude of Nusselt number remains nearly the same for the same plunge velocity. This phenomenon is observed for all the $k h$ values investigated. However, as $k h$ increases, the variations amongst different combinations of $k$ and $h$ increase. At low values of $k h \leq 1$, the flow is periodic and ordered with regular vortices being shed during the cycle. As $k h$ increases to 2 and 4, vortex strength increases and the flow enters into a chaotic regime in which non-linear vortex-vortex interactions start dominating the flow near the plate-fin and lead to marginally different heat transfer behavior at different $k$ and $h$ at the same $k h$ value. The differences in Nusselt number are more evident at the trailing half of the plate and could be a result of the variable interaction of TEVs with LEVs at different $k$ and $h$. 

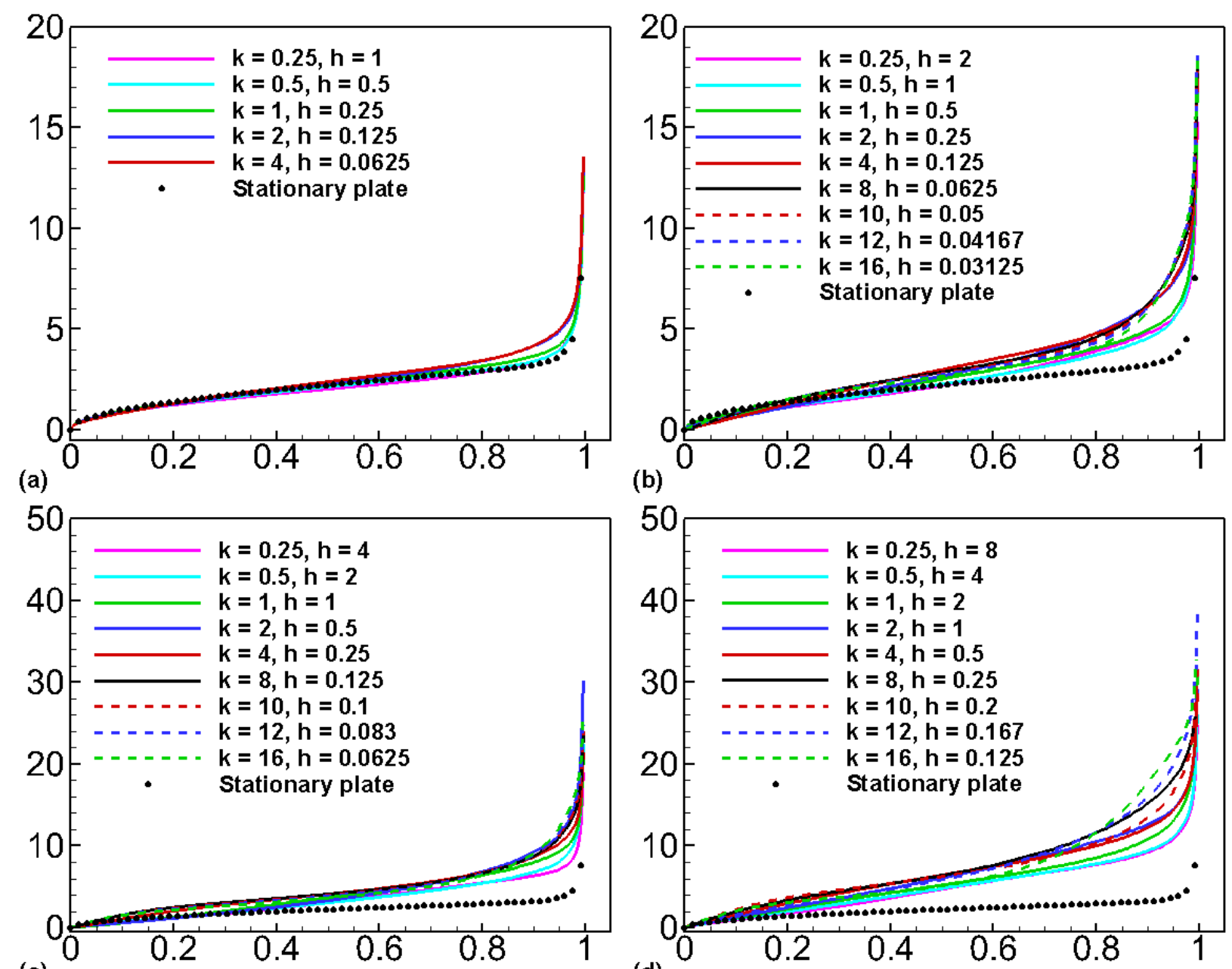

(c)

(b)

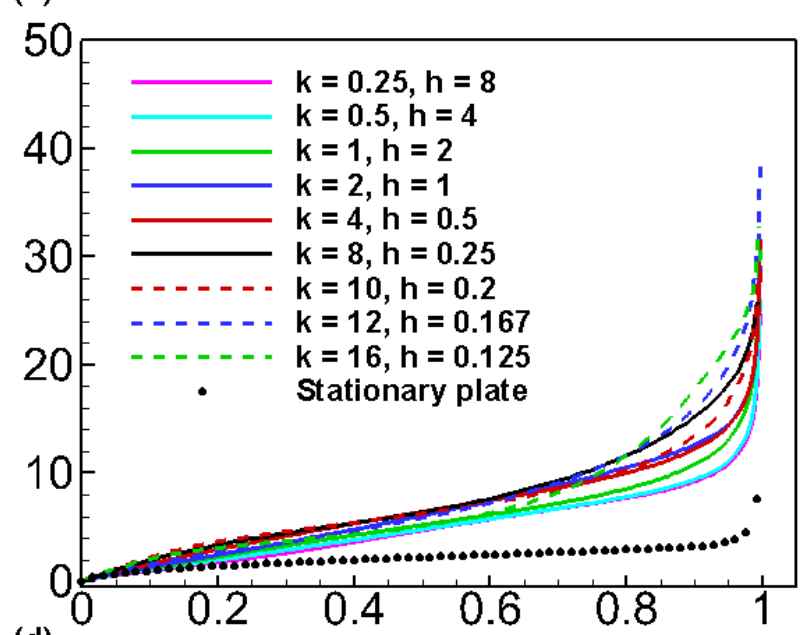

(d)

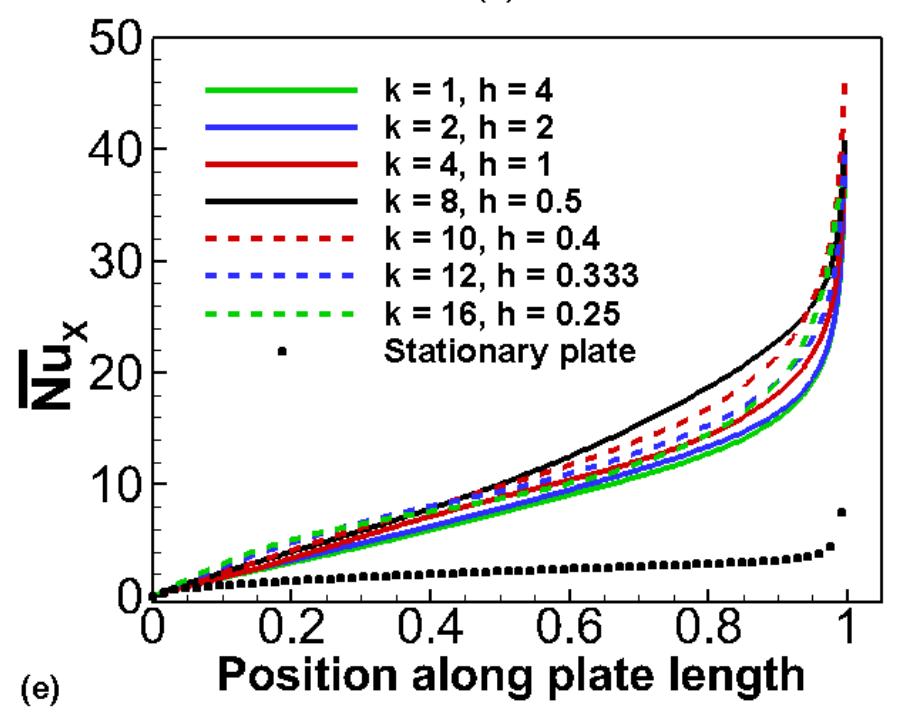

Figure 10: Nusselt number, $\overline{N u}_{x}$ distribution for cases with same plunge velocity. (a) $k h=0.25$, (b) $k h=0.5$, (c) $k h=1$, (d) $k h=2 \&$ (e) $k h=4$ 


\subsubsection{Heat Transfer Enhancement Factor}

The time averaged $\overline{N u}_{L}$ for the entire length of the plate is calculated for all the 39 cases mentioned in Table 1. From these values, $\overline{N u}_{k h}$ is calculated as the mean Nusselt number for all the cases with the same $k h$ value. An increasing trend of $\overline{N u}_{k h}$ with $k h$ is shown in Table 4. Also observed in Table 4 is that the standard deviation $(S D)$ of $\overline{N u}_{L}$ with the same $k h$ from $\overline{N u}_{k h}$ increases with increasing $k h$. The mean heat transfer enhancement factor expressed by Eqn. (16) is shown in Table 4.

$$
E F=\overline{N u}_{k h} / N u_{k h=0}
$$

At low $k h=0.25, E F \sim 1$ but as $k h$ increases $E F$ increases to a factor of 3 at $k h=4$.

Table 4: Heat transfer enhancement for different plunge velocities.

\begin{tabular}{ccccc}
\hline $\boldsymbol{k h}$ & $\overline{\boldsymbol{N u}}_{\boldsymbol{k h}}$ & $\boldsymbol{S D}$ & $\boldsymbol{E F}$ & \% Enhancement \\
\hline $\mathbf{0 . 0 0}$ & 6.398 & - & 1.0 & - \\
$\mathbf{0 . 2 5}$ & 6.230 & 0.182 & 0.97 & $-2.7 \%$ \\
$\mathbf{0 . 5}$ & 6.622 & 0.276 & 1.04 & $3.5 \%$ \\
$\mathbf{1 . 0}$ & 9.066 & 1.382 & 1.42 & $41.7 \%$ \\
$\mathbf{2 . 0}$ & 13.585 & 2.077 & 2.12 & $112.3 \%$ \\
$\mathbf{4 . 0}$ & 19.936 & 2.229 & 3.12 & $211.6 \%$ \\
\hline
\end{tabular}




\subsubsection{Behavioral Trend of Nusselt Number}

The present study, encompassing a wide range of frequencies ( $k$ ranging from 0.25 to 16 ) and amplitudes ( $h$ ranging from 0.03125 to 8 ), reveals that the Nusselt number increases monotonically with increasing $k h$. The behavioral trend of Nusselt number with increasing $k$ and $h$ is shown in Figure 11 in which the Nusselt number is plotted against plunge velocity $(k h)$ for constant $k$ (Fig. 11-a) and versus $k$ for constant $k h$ (Fig. 11-b). As can be seen from Figure 11-a, for all the frequencies $(k)$ tested, $\overline{N u}_{L}$ increases monotonically for increasing $h$ or $k h$. Irrespective of the individual frequency $(k)$ or amplitude $(h)$, low plunge velocity oscillations at $k h=0.25$ produce no enhancement of $N u$ when compared to the stationary plate $\left(\overline{N u}_{L}=6.398\right)$. Nominal enhancement occurs at $k h=0.5$. It is only after $k h \geq 1$ that there is substantive enhancement of $\overline{N u}_{L}$. As the amplitude or plunge velocity gets larger the increasing trend in $\overline{N u}_{L}$ persists but the rate of increment slows down for larger $k h$ values $(k h=2$ and 4$)$.

The variation of $N u$ with $k$ (or $h$ ) for a constant $k h$ is shown in Figure 11-b. As mentioned earlier, for a constant plunge velocity $(\mathrm{kh})$ irrespective of the individual combination of frequency and amplitude, similar order of heat transfer enhancement is observed. Although all the $\overline{N u}_{L}$ values for a constant plunge velocity are comparable, figure 11-b shows that there is a nominal variation in the $\overline{N u}_{L}$ values based on different combinations of frequency and amplitude. As frequency $(k)$ increases [i.e. amplitude $(h)$ decreases], $\overline{N u}_{L}$ for a constant $k h$ increases until a certain frequency value $(k=8)$ and then decreases marginally for higher $k$ values. 


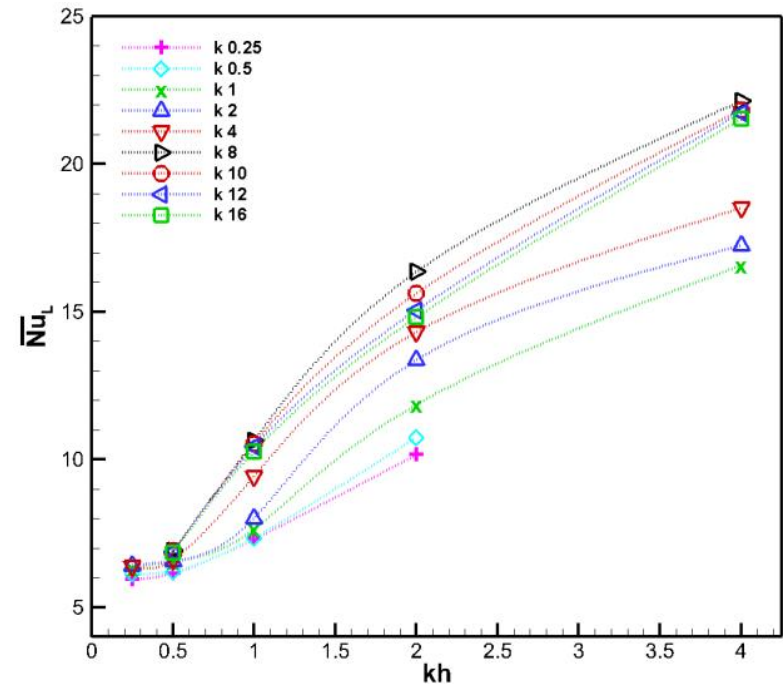

(a)

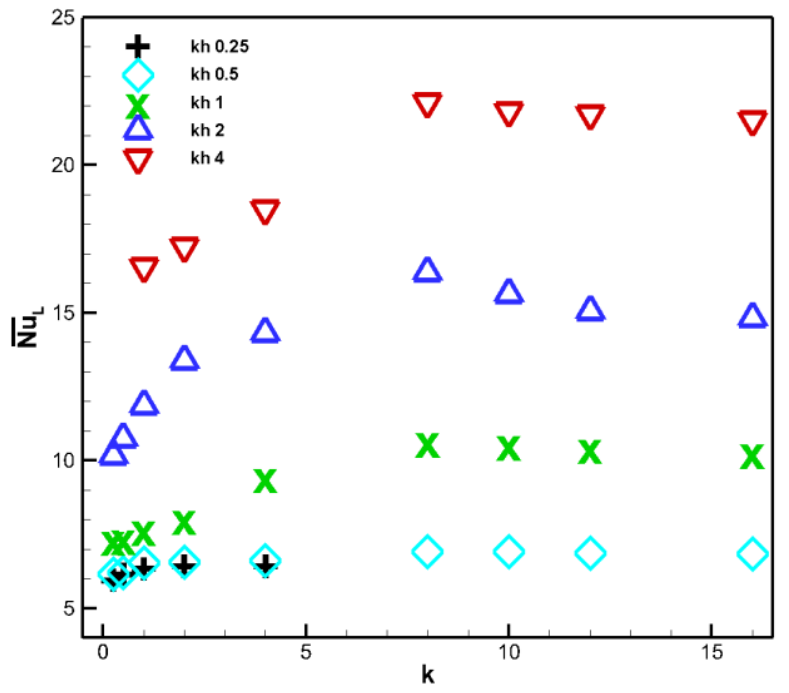

(b)

Figure 11: Variation of Nusselt number with (a) varying plunge velocities $(k h)$ for constant reduced frequencies $(k)$; (b) with varying reduced frequencies $(k)$ for constant plunge velocities (kh) 


\subsubsection{Energy Advantage of Vibrating Plate-Fin}

The enhancement in heat transfer at high $k$ or $h$ is comparable to and surpasses many other heat transfer augmentation methods. In most methods, heat transfer enhancement is accompanied by pressure loss and pumping power penalty. In contrast, in the current configuration, the vibrating plate not only has a zero drag penalty but adds energy to the flow for most values of $k h$. The thrust (negative drag) coefficient $\left(C_{T}\right)$ as defined in Eqn. 17, where $\bar{T}$ is the time-averaged thrust force, is shown in Figure 12 in which a negative $C_{T}$ indicates net drag on the fluid.

$$
C_{T}=\frac{\bar{T}}{\frac{1}{2} \rho U_{\infty}^{2} c}
$$

Drag is produced for $k h<0.5$ but at high reduced frequency only thrust is produced. It is noted that while the Nusselt number increases monotonically with $k h$, the thrust reaches a maximum at $k h=1$ and then decreases as $k h$ increases further. This is because whilst both LEVs and TEVs contribute to perturbing the thermal boundary layer and increasing the heat flux at the plate surface, only LEVs contribute to thrust whereas TEVs contribute to drag as shown in Figure 8. As $k h$ increases, the strength of both LEVs and TEVs increase, with a corresponding strengthening of vortex-vortex interactions. The outcome of these non-linear interactions at high $k h$ is that TEVs rather than convecting into the wake are drawn upstream in the vicinity of the plate surface, and LEVs are drawn away, both of which reduce thrust production, while still contributing to heat transfer augmentation. Thus heat transfer augmentation increases monotonically with $k h$ whereas thrust does not. However, the effect of LEVs being induced away 
from the plate surface at high $k h$ and $k$ results in a slow down or decrease in the Nusselt numbers as indicated in Figures 11-a and 11-b, respectively.

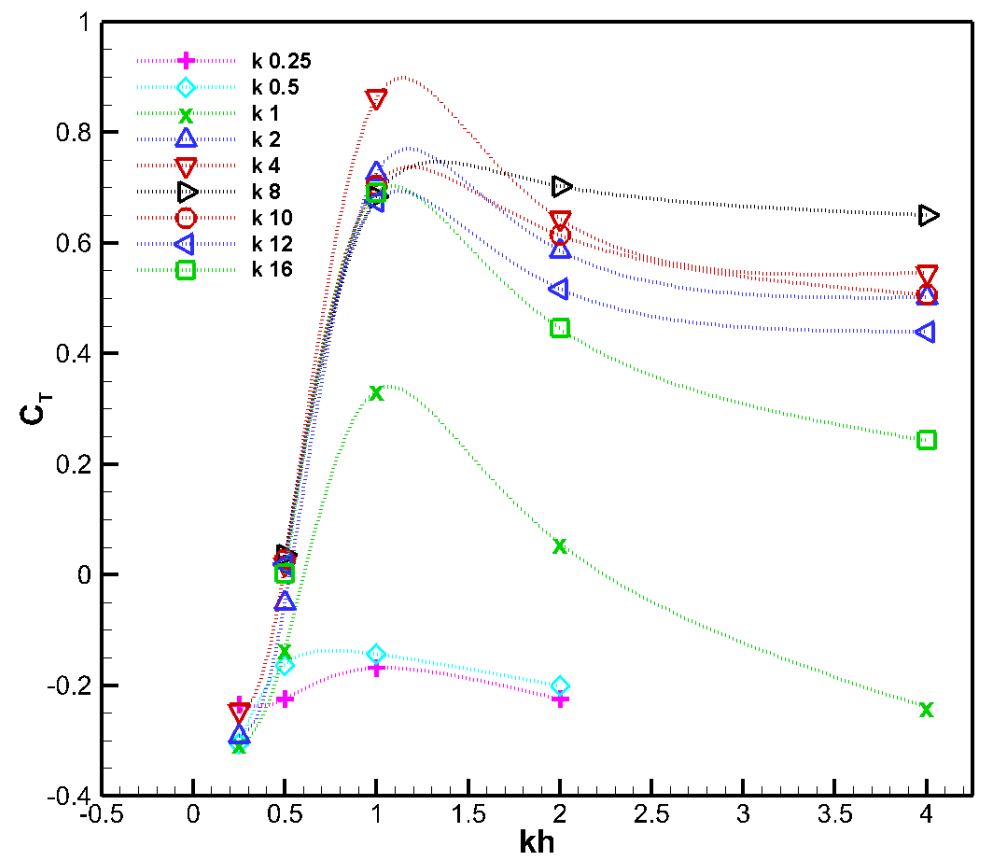

Figure 12: Variation of thrust coefficient with varying plunge velocities $(k h)$ for constant reduced frequencies $(k)$ 


\subsection{Parameterization of Nusselt Number}

In order to characterize the heat transfer augmentation achieved via vibrating a plate-fin, a parameterization of Nusselt number is done with various vibrating parameters. Figure 13(a-c) plots the dependence of $\overline{N u}_{L}$ on $k, h$ and $k h$ together with correlations which describe the dependence at different levels of fidelity. As shown previously, $\overline{N u}_{L}$ has the strongest correlation with $k h-$ as $k h$ increases, $\overline{N u}_{L}$ also increases monotonically. In comparison, $\overline{N u}_{L}$ has a weak dependence on both $k$ and $h . \overline{N u}_{L}$ decreases at low $h$ but the variation with $h$ gets weaker as $h$ increases. At low $k h, \overline{N u}_{L}$ increases with $k$ but also becomes fairly independent of $k$ as it increases beyond $k=8$. Table 5 summarizes the correlations and their goodness of fit with the numerical data. Eqn. (18) in Table 5 which admits non-linear variations in $k$ and $h$ exhibits the best fit. Eliminating the dependence on $k$ and $k^{2}$ in Eqn. (19) does not influence the goodness of fit measures substantially. Further eliminating the dependence of $h$ and $h^{2}$ in Eqn. (20) and (21) has a larger effect on the goodness of fit measures.
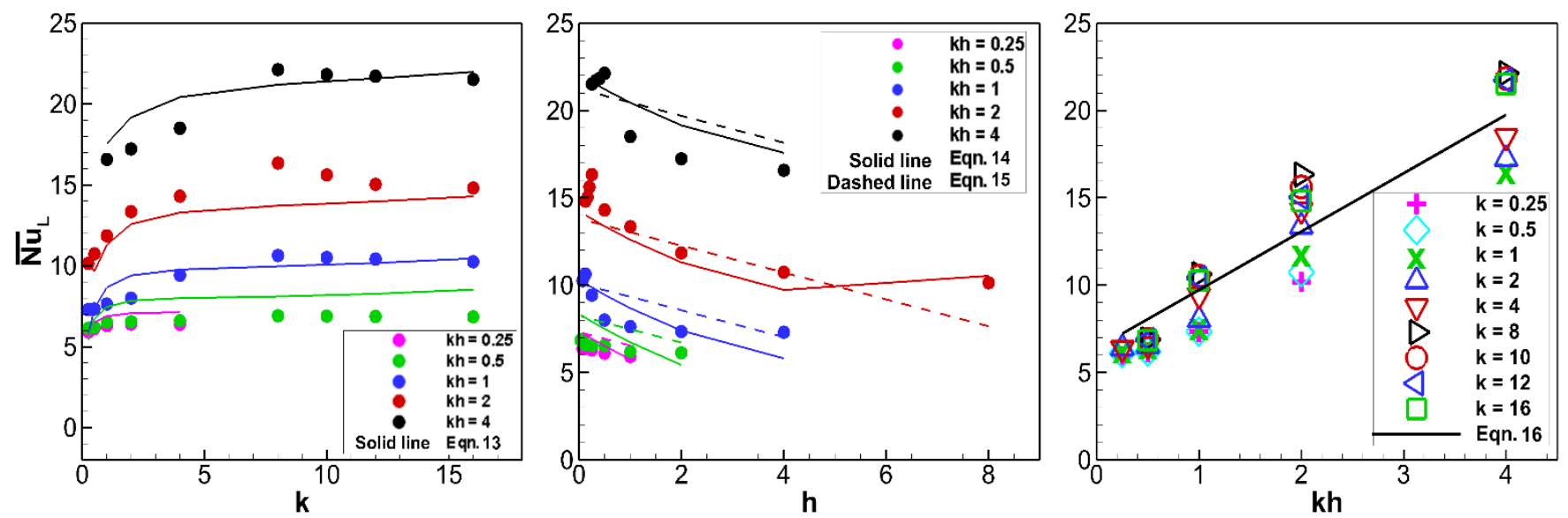
Figure 13: Parameterization of Nusselt number $\left(\overline{N u}_{L}\right)$ dependence on reduced frequency, $k$, plunge amplitude, $h$ and plunge velocity, $k h$ for $R e=100$ and $P r=0.71$ through Eqns. (18), (19), (20) and (21)

Table 5: Summary statistics of the goodness of fit of different correlations

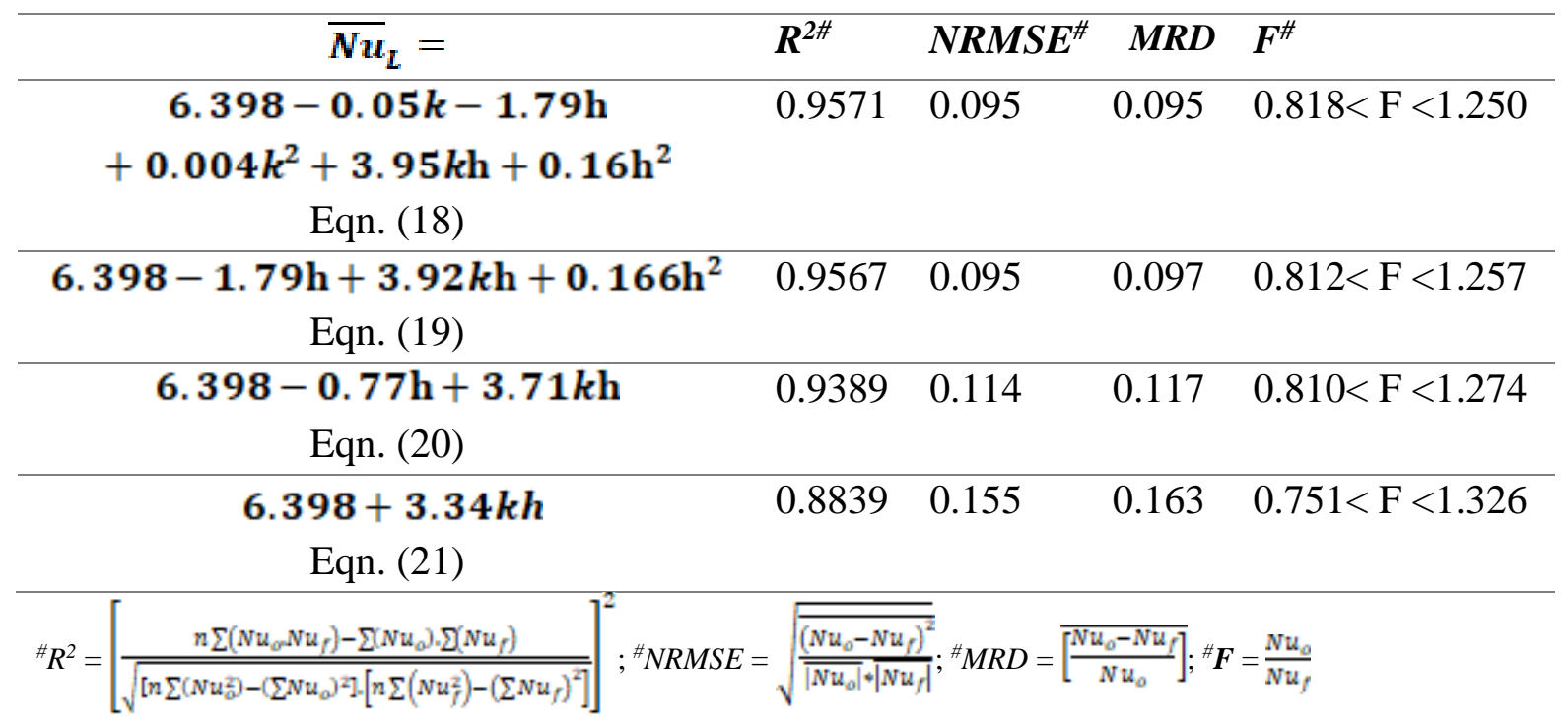




\section{Summary and Conclusions}

An infinitesimally thin oscillating plate-fin in an approach flow is investigated numerically to characterize the heat transfer at $R e=100$ and $P r=0.71$. The characteristic parameters of oscillation i.e. reduced frequency and plunge amplitude are varied as $0.25 \leq k \leq 16$ and $0.03125 \leq$ $h \leq 8$ to give plunge velocities ranging from $0.25 \leq k h \leq 4$, encompassing a wide range of length and time scales. The flow is characterized by vortex formation and shedding from the leading and trailing edge of the plate-fin as it oscillates, which continually destroy and replenish the fluid near the plate-fin surface leading to heat transfer enhancement. It is found that the combined effect of reduced frequency and plunge amplitude on heat transfer manifests in the distinct parameter of plunge velocity $(k h)$. It is also found that Nusselt number $(N u)$ increases monotonically with increasing $k h$. Irrespective of the individual frequency $(k)$ or amplitude $(h)$, low plunge velocity oscillations at $k h=0.25$ produce no enhancement of Nusselt number when compared to the stationary plate. While nominal enhancement occurs at $k h=0.5$, it is only after $k h \geq 1$ that a substantive enhancement of $N u$ is observed. As the plunge velocity gets larger the increasing trend in $\mathrm{Nu}$ persists but the rate of increment slows down for larger $k h$ values $(k h=2$ and 4).

A linear parameterization of Nusselt number with plunge velocity is established which eventually makes estimation of heat transfer enhancement based on the characteristic oscillation parameters much simpler. The work performed in this study is at a single Reynolds number of 100 in air for an idealized canonical geometry of an oscillating flat plate. While the results from this study cannot be directly applied to various fin configurations, this study finds relevance in practical applications where an enhancement in heat transfer is sought in regular as well as micro 
fin heat exchangers. Almost all systems have some extent of mechanical vibrations associated with them which would lead to high frequency oscillations of components. In scenarios where the mechanical vibration is limited by either the frequency or the amplitude owing to structural constraints, a variation in the parameter ' $k h$ ' as a whole could still result in increased heat transfer rates. Given the inherent vibration in a system, it is possible to use that vibration to enhance the thermal performance. It will be irrelevant if the vibration is frequency dominated or amplitude dominated, as long as the $k h$ value is significant, an elevated system performance should be expected. 


\section{References}

[1] Y. Liu, J. Duan, X. He, Y. Wang, Experimental investigation on the heat transfer enhancement in a novel latent heat thermal storage equipment, Appl. Therm. Eng. 142 (2018) 361-370. doi:10.1016/j.applthermaleng.2018.07.009.

[2] L. Jiang, S. Jiang, W. Cheng, Y. Nian, R. Zhao, Experimental study on heat transfer performance of a novel compact spray cooling module, Appl. Therm. Eng. 154 (2019) 150-156. doi:10.1016/j.applthermaleng.2019.03.078.

[3] D. P Haack, K. R Butcher, T. Kim, T. Lu, Novel Lightweight Metal Foam Heat Exchangers, Am. Soc. Mech. Eng. Process Ind. Div. PID. 6 (2001).

[4] M. Wong, I. Owen, C.J. Sutcliffe, A. Puri, Convective heat transfer and pressure losses across novel heat sinks fabricated by Selective Laser Melting, Int. J. Heat Mass Transf. 52 (2009) 281-288. doi:10.1016/j.ijheatmasstransfer.2008.06.002.

[5] N.I. Ibrahim, F.A. Al-sulaiman, S. Rahman, B.S. Yilbas, Z. Sahin, Heat transfer enhancement of phase change materials for thermal energy storage applications : A critical review, Renew. Sustain. Energy Rev. 74 (2017) 26-50. doi:10.1016/j.rser.2017.01.169.

[6] H. Yarmand, S. Gharehkhani, G. Ahmadi, S. Farid, S. Shirazi, S. Baradaran, E. Montazer, M. Nashrul, M. Zubir, M. Sadat, Graphene nanoplatelets - silver hybrid nanofluids for enhanced heat transfer, Energy Convers. Manag. 100 (2015) 419-428. doi:10.1016/j.enconman.2015.05.023.

[7] R.B. Ganvir, P. V Walke, V.M. Kriplani, Heat transfer characteristics in nano-fluid - A review, Renew. Sustain. Energy Rev. 75 (2017) 451-460. doi:10.1016/j.rser.2016.11.010.

[8] M.A. Elyyan, A. Rozati, D.K. Tafti, Investigation of dimpled fins for heat transfer enhancement in compact heat exchangers, Int. J. Heat Mass Transf. 51 (2008) 2950-2966. doi:10.1016/j.ijheatmasstransfer.2007.09.013.

[9] Y.F. Li, G.D. Xia, D.D. Ma, Y.T. Jia, J. Wang, Characteristics of laminar flow and heat transfer in microchannel heat sink with triangular cavities and rectangular ribs, Int. J. Heat Mass Transf. 98 (2016) 17-28. doi:10.1016/j.ijheatmasstransfer.2016.03.022.

[10] T. Alam, M. Kim, A comprehensive review on single phase heat transfer enhancement techniques in heat exchanger applications, Renew. Sustain. Energy Rev. 81 (2018) 813839. doi:10.1016/j.rser.2017.08.060. 
[11] A.E. Bergles, Recent developments in enhanced heat transfer, Heat Mass Transf. (2011) 1001-1008. doi:10.1007/s00231-011-0872-y.

[12] Z.Y. Guo, D.Y. Li, B. X. Wang, A novel concept for convective heat transfer enhancement, in: Proc. Symp. Energy Eng. 21st Century, Elsevier Ltd, 2000.

[13] L. Chai, A Review of Airside Heat Transfer Augmentation with Vortex Generators on Heat Transfer Surface, (2018). doi:10.3390/en11102737.

[14] M. Arulprakasajothi, K. Elangovan, K.H.C. Reddy, S. Suresh, Experimental investigation on heat transfer effect of conical strip inserts in a circular tube under laminar flow, Front. Energy. 10 (2016) 136-142. doi:10.1007/s11708-015-0389-z.

[15] M. Sheikholeslami, D.D. Ganji, Turbulent heat transfer enhancement in an air-to-water heat exchanger, Proc IMechE Part E J Process Mech. Eng. 231 (2017) 1235-1248. doi:10.1177/0954408916664758.

[16] S. Alekseenko, Transfer Processes in Vortex Flow, in: Int. Heat Transf. Conf. Digit., 2014: pp. 155-172. doi:10.1615/IHTC15.kn.000008.

[17] J. Liu, G. Xie, T.W. Simon, Turbulent flow and heat transfer enhancement in rectangular channels with novel cylindrical grooves, Int. J. Heat Mass Transf. 81 (2015) 563-577. doi:10.1016/j.ijheatmasstransfer.2014.10.021.

[18] N. Zheng, P. Liu, F. Shan, Z. Liu, W. Liu, Heat transfer enhancement in a novel internally grooved tube by generating longitudinal swirl flows with multi-vortexes, Appl. Therm. Eng. 95 (2016) 421-432. doi:10.1016/j.applthermaleng.2015.11.066.

[19] O. Rezaei, O. Ali, A. Marzban, D. Toghraie, F. Pourfattah, The numerical investigation of heat transfer and pressure drop of turbulent flow in a triangular microchannel, Phys. E. 93 (2017) 179-189. doi:10.1016/j.physe.2017.06.013.

[20] M.E. Steinke, S.G. Kandlikar, Single-Phase Heat Transfer Enhancement Techniques in Microchannel and Minichannel Flows, ICMM2004-2328. (2015).

[21] X. Wei, Y. Han, J. Quan, A novel wavy-tape insert configuration for pipe heat transfer augmentation, Energy Convers. Manag. 127 (2016) 140-148. doi:10.1016/j.enconman.2016.09.006.

[22] K. Yakut, B. Sahin, Flow-induced vibration analysis of conical rings used for heat transfer enhancement in heat exchangers, Appl. Energy. 78 (2004) 273-288. doi:10.1016/j.apenergy.2003.09.001. 
[23] M. Tian, L. Cheng, Y. Lin, G. Zhang, Heat Transfer Enhancement by Crossflow-Induced Vibration, Heat Transf. Res. 33 (2004) 211-218. doi:10.1002/htj.20012.

[24] J. Shi, J. Hu, S.R. Schafer, C.C.L. Chen, Numerical study of heat transfer enhancement of channel via vortex-induced vibration, Appl. Therm. Eng. 70 (2014) 838-845. doi:10.1016/j.applthermaleng.2014.05.096.

[25] X. Sun, Z. Ye, J. Li, K. Wen, H. Tian, Forced convection heat transfer from a circular cylinder with a flexible fin, Int. J. Heat Mass Transf. 128 (2019) 319-334. doi:10.1016/j.ijheatmasstransfer.2018.08.123.

[26] K. Li, X. Xu, Z. Li, H. Chen, Y. Chen, S. Wang, Z. Ke, G. Huang, C. Chen, C. Chen, Airside heat transfer enhancement in plate-fin channel with an airfoil-based self-agitator, Int. J. Heat Mass Transf. 128 (2019) 715-727. doi:10.1016/j.ijheatmasstransfer.2018.09.040.

[27] K. Li, S. Wang, Z. Ke, C. Chen, A novel caudal-fin-inspired hourglass-shaped selfagitator for air-side heat transfer enhancement in plate-fin heat exchanger, Energy Convers. Manag. 187 (2019) 297-315. doi:10.1016/j.enconman.2019.03.048.

[28] M. Sheikholeslami, M. Gorji-bandpy, D. Domiri, Review of heat transfer enhancement methods : Focus on passive methods using swirl fl ow devices, Renew. Sustain. Energy Rev. 49 (2015) 444-469. doi:10.1016/j.rser.2015.04.113.

[29] M. Sheikholeslami, M.M. Bhatti, Active method for nanofluid heat transfer enhancement by means of EHD, Int. J. Heat Mass Transf. 109 (2017) 115-122. doi:10.1016/j.ijheatmasstransfer.2017.01.115.

[30] M. Bahiraei, M. Hangi, Flow and heat transfer characteristics of magnetic nano fl uids : A review, J. Magn. Magn. Mater. 374 (2015) 125-138. doi:10.1016/j.jmmm.2014.08.004.

[31] M. Legay, N. Gondrexon, L. Person, Enhancement of Heat Transfer by Ultrasound : Review and Recent Advances, Int. J. OfChemical Eng. (2011). doi:10.1155/2011/670108.

[32] B. Loh, N. Carolina, P.I. Ro, C. Kleinstreuer, Acoustic streaming induced by ultrasonic flexural vibrations and associated enhancement of convective heat transfer, J. Acoust. Soc. Am. 111 (2002) 875-883. doi:10.1121/1.1433811.

[33] H. Kim, Y.G. Kim, Enhancement of Natural Convection and Pool Boiling Heat, in: ASME Summer Heat Transf. Conf., 2003: pp. 1-8. https://proceedings.asmedigitalcollection.asme.org.

[34] A. Klaczak, Report from experiments on heat transfer by forced vibrations of exchangers, 
Heat Mass Transf. 32 (1997) 477-480.

[35] C.J.B. R. E. Forbes, C. T. Carley, Vibration Effects on Convective heat Transfer in Enclosures, J. Heat Transfer. (1970).

[36] R. Lemlich, Effect of Vibration on Natural Convective Heat Transfer, Ind. Eng. Chem. (1955) 1175-1180. doi:10.1021/ie50546a024.

[37] C.B. Baxi, N.A. Ramachandra, Effect of Vibration on Heat Transfer From Spheres, Winter Annu. Meet. New York, N. Y. 1 (1968) 68.

[38] R. M. Fand, A Comparison of the Influence of Mechanical and Acoustical Vibrations on Free Convections From a Horizontal Cylinder The Influence of Temperature Difference on the Turbulent Forced-Convection Heating of Water, Trans. ASME. (1962) 268.

[39] M. Kimber, S. V Garimella, Local Heat Transfer Coefficient Induced by Piezoelectrically Actuated Vibrating Cantilevers, Trans. ASME. 129 (2007) 1168. doi:10.1115/1.2740655.

[40] D.H. Kim, Y.H. Lee, S.H. Chang, Effects of mechanical vibration on critical heat flux in vertical annulus tube, Nucl. Eng. Des. 237 (2007) 982-987. doi:10.1016/j.nucengdes.2006.11.002.

[41] A.R. K. Sreenivasan, Effect of Vibration on Heat Transfer from a Horizontal Cylinder to a Normal Air Stream, Int. J. Heat Mass Transf. 3 (1961) 60-67.

[42] K.E. K. Takahashi, A new correlation method for the effect of vibration on forcedconvection heat transfer, J. Chem. Eng. Japan. 23 (1990).

[43] L. Cheng, T. Luan, W. Du, M. Xu, International Journal of Heat and Mass Transfer Heat transfer enhancement by flow-induced vibration in heat exchangers, Int. J. Heat Mass Transf. 52 (2009) 1053-1057. doi:10.1016/j.ijheatmasstransfer.2008.05.037.

[44] H.S. Park, Thermal Performance Improvement of a Heat Sink with Piezoelectric Vibrating Fins, in: Proc. 14th Int. Heat Transf. Conf. IHTC14, 2010.

[45] A.R. Sarhan, M.R. Karim, Z.K. Kadhim, J. Naser, Experimental investigation on the effect of vertical vibration on thermal performances of rectangular flat plate, Exp. Therm. Fluid Sci. 101 (2019) 231-240. doi:10.1016/j.expthermflusci.2018.10.024.

[46] J.S. Go, Design of a microfin array heat sink using flow-induced vibration to enhance the heat transfer in the laminar flow regime, Sensors Actuators A. 105 (2003) 201-210. doi:10.1016/S0924-4247(03)00101-8.

[47] D.K. Tafti, GenIDLEST - A scalable parallel computational tool for simulating complex 
turbulent flows, in: ASME-IMECE, 2001: pp. 347-356.

[48] P. Gopalakrishnan, D.K. Tafti, A Parallel Multiblock Boundary Fitted Dynamic Mesh Solver for Simulating Flows with Complex Boundary Movement, 38th Fluid Dyn. Conf. Exhib. (2009) 1-20.

[49] S.Y. Kim, B.H. Kang, Flow and Heat Transfer Correlations for Porous Fin in a Plate-Fin Heat Exchanger, J. Heat Transfer. 122 (2000) 572-578.

[50] A. Soni, Study of Thermal Performance between Plate-fin, Pin-fin and Elliptical Fin Heat Sinks in Closed Enclosure under Natural Convection, Int. Adv. Res. J. Sci. Eng. Technol. 3 (2016) 133-139. doi:10.17148/IARJSET.2016.31126. 